\title{
Enhanced eosinophilic inflammation associated with antibody and complement-mediated pneumonic insults in severe COVID-19
}

\section{Dong-Min Kim}

Chosun University College of Medicine

Jun-Won Seo

Chosun University College of Medicine

\section{Yuri Kim}

Seoul National University College of Medicine Jooyeon Lee

Chungnam National University School of Medicine

\section{Uni Park}

Seoul National University College of Medicine

\section{$\mathrm{Na}$-Young $\mathrm{Ha}$}

Seoul National University College of Medicine Jaemoon Koh

Seoul National University College of Medicine Hyoree Park

Seoul National University College of Medicine Jae-Won Lee

Seoul National University College of Medicine Hyo-Jin Ro

Seoul National University College of Medicine $\mathrm{Na}$ Ra Yun

Chosun University College of Medicine

\section{Da Young Kim}

Chosun University College of Medicine

\section{Sung Ho Yoon}

Chosun University College of Medicine

\section{Yong Sub $\mathrm{Na}$}

Chosun University College of Medicine

\section{Do Sik Moon}

Chosun University College of Medicine

\section{Sung-Chul Lim}


Chosun University College of Medicine

\section{Choon-Mee Kim}

Chosun University College of Medicine

\section{Kyeongseok Jeon}

Seoul National University College of Medicine

\section{Jun-Gu Kang}

Jeonbuk National University

\section{Hyeongseok Jeong}

Chungnam National University School of Medicine

\section{Jungok Kim}

Chungnam National University School of Medicine

\section{Shinhyea Cheon}

Chungnam National University School of Medicine

\section{Kyung Mok Sohn}

Chungnam National University School of Medicine Jae Young Moon

Chungnam National University School of Medicine

\section{Sungmin Kym}

Chungnam National University Sejong Hospital

\section{Myung-Shin Lee}

Eulji University School of Medicine

\section{Hyun-Je Kim}

Samsung Advanced Institute for Health Sciences \& Technology

\section{Woong-Yang Park}

Samsung Advanced Institute for Health Sciences \& Technology

\section{Hyun-Woo Shin}

Seoul National University College of Medicine

\section{Hye-Young Kim}

Seoul National University College of Medicine

\section{Chung-Hyun Cho}

Seoul National University College of Medicine

\section{Yoon Kyung Jeon}

Seoul National University College of Medicine

\section{Yeon-Sook Kim}

Chungnam National University School of Medicine

\section{Nam-Hyuk Cho ( $\sim$ chonh@snu.ac.kr)}

Seoul National University College of Medicine 


\section{Research Article}

Keywords: COVID-19, SARS-CoV-2, eosinophilic inflammation, pneumonia

Posted Date: November 3rd, 2020

DOl: https://doi.org/10.21203/rs.3.rs-101027/v1

License: (c) (i) This work is licensed under a Creative Commons Attribution 4.0 International License. Read Full License 


\section{Enhanced eosinophilic inflammation associated with antibody and complement-mediated pneumonic insults in severe COVID-19} Dong-Min Kim ${ }^{1,13, *}$, Jun-Won Seo ${ }^{1,13}$, Yuri Kim ${ }^{2,3,413}$, Jooyeon Lee ${ }^{5,13}$, Uni Park ${ }^{2,3,13}$, Na-Young $\mathrm{Ha}^{2,3,4,13}$, Jaemoon $\mathrm{Koh}^{6,13}$, Hyoree Park ${ }^{2,3}$, Jae-Won Lee ${ }^{2,3}$, Hyo-Jin Ro ${ }^{2,3}$, Na Ra Yun ${ }^{1}$, Da Young Kim ${ }^{1}$, Sung Ho Yoon ${ }^{1}$, Yong Sub Na ${ }^{1}$, Do Sik Moon ${ }^{1}$, Sung-Chul Lim ${ }^{7}$, Choon-Mee Kim ${ }^{8}$, Kyeongseok Jeon ${ }^{2,3}$, Jun-Gu Kang ${ }^{9}$, Hyeongseok Jeong ${ }^{5}$, Jungok Kim ${ }^{10}$, Shinhyea Cheon ${ }^{5}$, Kyung Mok Sohn ${ }^{5}$, Jae Youg Moon ${ }^{10}$, Sungmin Kym ${ }^{10}$, Myung-Shin Lee ${ }^{11}$, Hyun-Je Kim ${ }^{12}$, Woong-Yang Park ${ }^{12}$, Hyun-Woo Shinn ${ }^{3}$, Hye-Young Kim ${ }^{3}$, Chung-Hyun Cho ${ }^{3}$, Yoon Kyung Jeon ${ }^{6}$, Yeon-Sook Kim ${ }^{5, *}$, and Nam-Hyuk $\mathrm{Cho}^{2,3,4, *}$

${ }^{1}$ Department of Internal Medicine, Chosun University College of Medicine, Gwangju, Republic of Korea.

${ }^{2}$ Department of Microbiology and Immunology, ${ }^{3}$ Department of Biomedical Sciences, Seoul National University College of Medicine, Seoul, Republic of Korea.

${ }^{4}$ Institute of Endemic Disease, Seoul National University Medical Research Center and Bundang Hospital, Seoul, Republic of Korea.

${ }^{5}$ Department of Internal Medicine, Chungnam National University School of Medicine, Daejeon, Republic of Korea.

${ }^{6}$ Department of Pathology, Seoul National University College of Medicine, Seoul, Republic of Korea

${ }^{7}$ Department of Pathology, ${ }^{8}$ Premedical Science, Chosun University College of Medicine, 
23 Gwangju, Republic of Korea.

$24{ }^{9}$ Korea Zoonosis Research Institute, Jeonbuk National University, Iksan, Republic of Korea.

25

26

27

28

29

30

31

32

33

34

35

36

37

38

39

40

41

42

43

4

5

${ }^{10}$ Department of Internal Medicine, Chungnam National University Sejong Hospital, Sejong, Republic of Korea.

${ }^{11}$ Department of Microbiology and Immunology, Eulji University School of Medicine, Daejeon, Republic of Korea.

${ }^{12}$ Department of Health Sciences and Technology, Samsung Advanced Institute for Health Sciences \& Technology, Sungkyunkwan University, Seoul, South Korea.

${ }^{13}$ These authors contributed equally.

*Correspondence: Dong-Min Kim (drongkim@chosun.ac.kr), Yeon-Sook Kim (alice@cnuh.co.kr), and Nam-Hyuk Cho (chonh@snu.ac.kr)

6

7

8

9

0

1




\section{$44 \quad$ Abstract}

Despite the worldwide effect of the Coronavirus disease 2019 (COVID-19) pandemic, the underlying mechanisms of fatal viral pneumonia remain elusive. Here, we conducted kinetic profiling of respiratory leukocytes and associated inflammatory mediators to show that severe COVID-19 is associated with delayed but enhanced eosinophilic inflammation when compared to mild cases. In addition, we confirmed increased Th2-biased adaptive immune responses, accompanying overt complement activation, in the severe group. Moreover, enhanced antibody responses and complement activation was associated with disease pathogenesis as evidenced by formation of immune complexes and membrane attack complexes in airways and vasculature of lung biopsies from a fatal case, as well as by enhanced hallmark gene set signatures of Fc $\gamma \mathrm{R}$ signaling and complement activation in myeloid cells of respiratory specimens from severe COVID-19 patients. We also observed expression of viral antigen in lung epithelial and endothelial cells without producing viruses during late stage of COVID-19, indicating abortive viral infection which may further fuel antibody responses and aggravate immune-complexmediated inflammation. These results suggest that SARS-CoV-2 infection may drive scripted specific innate immune responses, including eosinophilic inflammation, and subsequent pulmonary pathogenesis via enhanced Th2-biased immune responses, which might be crucial drivers of severe pneumonia in COVID-19 patients.

Keywords: COVID-19, SARS-CoV-2, eosinophilic inflammation, pneumonia 


\section{Introduction}

67 Severe acute respiratory syndrome coronavirus-2 (SARS-CoV-2) ${ }^{1}$ has been rapidly spreading worldwide since Dec. 2019 with an average mortality rate of approximately 3.2\% (https://covid19.who.int). The primary cause of disease fatality is severe pneumonia, resulting in acute respiratory syndrome (ARDS). ${ }^{2}$ Around $80 \%$ of confirmed cases are asymptomatic or have mild symptoms, including fever, cough, sore throat, and myalgia, whereas the rest often develop severe pneumonia requiring supplemental oxygen therapy. ${ }^{3,4}$ The most common finding of radiological imaging is bilateral, ground-glass opacity in the periphery of the lungs. ${ }^{4}$ The mechanisms underlying this varying degree of pneumonia severity observed in COVID-19 patients remain elusive. ${ }^{5}$ In particular, the dynamics of pathologic inflammation and the central culprits of pneumonic progression leading to severe ARDS and death still remain unclear, despite numerous studies profiling systemic immune signatures. ${ }^{6,7}$

In order to characterize the pathogenic hallmarks of severe pneumonia in COVID-19 patients, we performed kinetic analysis of inflammatory features of specimens collected from confirmed patients with various degrees of clinical symptoms. We systematically analyzed inflammatory components and leukocytes in bronchoalveolar lavage fluids (BALFs), sputa, lung tissue biopsies, and bloods to characterize kinetic responses of pulmonary inflammation upon viral infection. This extensive analysis revealed that severe COVID-19 is associated with enhanced eosinophilic pulmonary inflammation, as identified by cytological analysis and detection of granular contents derived from the inflammatory cells. In addition, kinetic profiling of inflammatory mediators, including various cytokines and chemokines, and titration of antibodies against a viral antigen revealed emerging Th2-biased adaptive immune responses, coupled to 
overt complement activation, especially in the severe group. In addition, we observed extensive immune complexes and membrane attack complexes in pulmonary airways and vasculatures of lung biopsies from a fatal case. Moreover, persistent expression of viral antigens, potentially via abortive infection, in inflamed lungs at late stage may irreversibly aggravate antibody and complement-mediated pulmonary damage in fatal COVID-19. These results suggest that SARSCoV-2 infection may drive scripted specific innate immune responses, including eosinophilic inflammation, and subsequent Th2-biased antigen-specific immune responses, which may contribute to COVID-19 associated severe pneumonia.

\section{Results and Discussion}

Baseline characteristics of the confirmed patients included in this study are summarized in Supplementary Table S1 and Fig. S1. The mild group includes 36 patients who were asymptomatic, with mild respiratory symptoms but no detectable pneumonia, or with mild to moderate pneumonia determined by chest imaging and clinical symptoms. The severe group includes 16 patients who suffered from severe pneumonia requiring high flow oxygen supply and/or mechanical ventilation. All the patients in the severe group survived and were discharged, except one patient (P15) who succumbed to death due to fatal ARDS. The patients were also divided into two sets: Group 1 includes 15 patients (10 mild and 5 severe group patients) who provided blood and respiratory specimens at different time points after symptoms onset. Group 2 includes 37 patients (26 mild and 11 severe patients) who provided respiratory specimens during the acute phase of COVID-19.

First, we investigated the potential association of viral loads of respiratory secretions with 
110 systemic inflammation, as indicated by the levels of C-reactive proteins (CRP) in plasma. ${ }^{8}$ The 111 kinetics of viral loads in upper (nasopharyngeal and throat swabs) and lower (sputa and BALFs) 112 respiratory tracts were not significantly different between severe and mild groups (Fig. 1a), as 113 previously reported. ${ }^{9,10}$ However, the levels of CRP in plasma were more significantly elevated 114 in severe patients, especially during the first 20 days after symptom onset and peaking around 115 day 10 (D10) (Fig. 1b). Therefore, viral loads measured in respiratory secretions may not be 116 significantly associated with disease severity and systemic inflammation in COVID-19 patients.

117 To investigate the potential causative factors driving severe pulmonary inflammation during the acute phase of COVID-19, we tried to directly analyze the inflammatory cells and mediators in respiratory specimens. 45 BALFs and sputa samples collected from patient groups 1 and 2 at various time points after symptom onset were analyzed using H\&E stained cytologic specimens to identify types and proportions of immune cell subsets infiltrating infected lungs (Fig. 2a and 122 Supplementary Table S2). Despite wide variation among the specimens, BALFs and sputa from COVID-19 patients mainly contained PMNs (mostly neutrophils, mean \pm S.D.: $46.8 \pm 34.3 \%$ in 124 total leukocytes), monocytes/macrophages $(37.5 \pm 32.7 \%)$, and a few lymphocytes $(11.6 \pm$ $12512.2 \%)$. We also observed 48.9\% (22/45) of the respiratory specimens included eosinophils (4.3 $126 \pm 7.3 \%$ ) (Fig. 2a and Supplementary Table S2). When we compared the level of each 127 inflammatory cell type between mild and severe patients, the relative proportion of all the cell types were not significantly different between the groups, although eosinophils were slightly 129 higher in the mild group $(6.0 \pm 9.4 \%)$ than severe patients $(2.8 \pm 4.6 \%, p=0.18)$ (Supplementary 130 Table S2).

131 In order to characterize the immune cell population in detail and compare them with systemic 132 leukocytes in COVID-19 patients, five paired BALFs and blood leukocytes collected from three 
severe patients at the indicated day after symptom onset were directly applied for flow cytometric analysis. We identified the relative proportions of myeloid cells, including CD14 monocytes/macrophages and $\mathrm{SSC}^{\mathrm{High}} / \mathrm{CD} 14^{-} \mathrm{PMNs}$, among BALFs' and blood leukocytes, as well as $\mathrm{CD}^{+} \mathrm{T}$ cells, CD20 ${ }^{+} \mathrm{B}$ cells, $\mathrm{CD}^{+} / \mathrm{CD}^{-} 6^{-}$natural killer $(\mathrm{NK})$ cells, and $\mathrm{CD}^{+} / \mathrm{CD}^{+} 6^{+}$ NKT-like (denoted as NKT hereafter) cells, ${ }^{11}$ based on expression levels of the indicated surface markers (Fig. 2b, Supplementary Fig. S2 - S5). In addition, PMNs were further defined by assessing the surface expression of CD16 and CD24. ${ }^{12} \mathrm{~T}$ cells and NKT cells were also characterized by measuring CD4 and CD8 surface expression. As we observed in respiratory specimens by $H \& E$ staining, PMNs were the predominant inflammatory cells, ranging from $45.5 \%$ to $79.7 \%$ of lung-infiltrating leukocytes (Fig. $2 \mathrm{~b}$ and Supplementary Fig. S3). This was followed by lymphoid cells, comprising $9.9 \%$ to $34.0 \%$, and $\mathrm{CD}_{14}^{+}$monocytes/macrophages, accounting for $2.8 \sim 11.1 \%$. Among the PMNs in BALFs of severe patients, neutrophils were generally dominant in the inflamed lungs $\left(36.3 \% \sim 62.1 \%\right.$ among CD45 $5^{+}$leukocytes $)$and $1.2 \sim$ $36.9 \%$ of leukocytes were eosinophils. It is notable that eosinophils were particularly elevated at $36.9 \%$ of total leukocytes in a BALF specimen from the fatal case P15 at D30. Among the lymphocyte population in BALFs from the severe cases, T cells were the dominant cell type, accounting for $72.3 \sim 89.6 \%$, whereas $\mathrm{B}$ cells were barely detected $(0.4 \sim 1.9 \%)$ (Fig. 2 b, Supplementary Fig. S4). NK and NKT cells represented $2.3 \sim 9.6 \%$ and $3.7 \sim 11.5 \%$ of pulmonary lymphocytes, respectively. The ratio of $\mathrm{CD}^{+}$and $\mathrm{CD}^{+} \mathrm{T}$ cell in the respiratory specimens fluctuated (1.6 at D14, 0.6 at D20, 1.1 at D27, 1.0 at D30, and 1.9 at D39), but CD8 ${ }^{+}$ $\mathrm{T}$ cells generally predominated, suggesting a potential role of cytotoxic $\mathrm{T}$ cell responses in the respiratory tract of severe pneumonia (Fig 2b: right panel, and Supplementary Fig. S5). It is interesting to note that the majority of pulmonary NKT cells were CD8-positive (Supplementary 
Fig. S5).

To assess the kinetic changes of immune cell types in respiratory specimens of COVID-19 patients, the relative proportion of each cell type was measured over time after symptom onset by H\&E staining and flow cytometry (Fig. 2c and d). Despite individual variations and fluctuations among the specimens, PMNs, primarily neutrophils, were sustained in respiratory specimens from severe cases, but rapidly declined in mild patients during the first 20 days after symptom onset. Monocytes/macrophages also gradually decreased after an initial peak around D10 after symptom onset in both mild and severe groups (Fig. 2c), whereas lymphocytes gradually increased with more rapid response in mild patients than the severe group (Fig. 2d). Interestingly, eosinophils were detectable in 55\% (11/20) of specimens from mild patients during the first 10 days after symptom onset, whereas their infiltration was rather delayed and peaked during D1020 in severe cases (Fig. 2d). Eosinophil-positive rate in severe patients' sample was 64.0\% (16/25) when assessed by H\&E and flow cytometry.

Since we observed pulmonary infiltration of PMNs, including neutrophils and eosinophils, we measured the levels of inflammatory mediators derived from neutrophils (lipocalin-2, LCN; calprotectin, CAPL) ${ }^{13,14}$ and eosinophils (eosinophil-derived neurotoxin, EDN; eosinophilic cationic protein, ECP $)^{15,16}$ in the respiratory specimens to assess innate cellular activation. $\mathrm{LCN}$ and CALP were detected in specimens from both mild and severe patients (Fig. 3a). The relative levels of both mediators were not significantly different between mild (mean \pm S.D.: $977.8 \pm$ $1366.4 \mathrm{ng} / \mathrm{ml}$ and $155.3 \pm 478.7 \mathrm{mg} / \mathrm{ml}$ for LCN and CALP, respectively) and severe groups $(905.6 \pm 1741.7 \mathrm{ng} / \mathrm{ml}$ and $44.7 \pm 91.1 \mathrm{mg} / \mathrm{ml}$ for LCN and CALP, respectively), although neutrophil responses were more sustained in severe cases (Fig. 3a). In contrast, levels of EDN and ECP were generally higher in the respiratory specimens from severe patients (mean \pm S.D.: 
$937.5 \pm 1244.7 \mathrm{pg} / \mathrm{ml}$ and $60.5 \pm 77.5 \mathrm{ng} / \mathrm{ml}$ for EDN and ECP, respectively) than mild cases $(231.8 \pm 375.6 \mathrm{pg} / \mathrm{ml}$ and $32.5 \pm 62.3 \mathrm{ng} / \mathrm{ml}$ for EDN and ECP, respectively) (Fig. 3b). Of note, the levels of ECP were significantly higher in severe cases than the mild group $(p=0.0187)$. In addition, the level of mast cell tryptase (MCT) derived from mast cells upon activation was approximately 3.5 times higher in the severe group $(31.1 \pm 42.3 \mathrm{ng} / \mathrm{ml})$ than mild cases $(8.8 \pm 4.4$ $\mathrm{ng} / \mathrm{ml}$ ), although the difference was not statistically significant $(p=0.1141)$ (Fig. 3c). We also examined macrophage activation syndrome by measuring soluble CD163 (sCD163) molecules in the respiratory samples. ${ }^{17,18}$ The levels of sCD163 peaked at around D10 in both mild and severe groups (Fig. 3d). The peak response was higher in severe cases than the mild group and the overall levels were significantly enhanced in severe patients, as previously suggested. ${ }^{18}$ This confirms the role of macrophage infiltration and activation, potentially towards M2 phenotype, during the early phase of severe COVID-19 pneumoina. ${ }^{19}$ Nevertheless, the concentration of sCD163 in severe cases after the initial peak response was similar to those of mild cases, suggesting a particularly intense contribution during the early phase of pneumonia progression. In addition, cytotoxic activity of CTLs and NK cells was not significantly different between mild and severe groups, when assessed by measuring granzyme A in respiratory specimens (Fig. 3e). Therefore, these results highlight the significant contribution of enhanced and sustained anaphylactic inflammation driven by infiltrating eosinophils in severe COVID-19 pneumonia, ${ }^{20}$ together with an acute spike in macrophage activation during the early phase. ${ }^{17}$

To further define pulmonary inflammation associated with COVID-19 patients, we examined multiple cytokines, chemokines, and inflammatory mediators in 31 respiratory samples, including sputa and BALFs, collected from 11 patients (6 mild cases and 5 severe cases in group 1) at various time points after symptom onset (Fig. 4, Supplementary Table S3 and Fig. S6). 
When we assessed the correlation of the overall levels of theses soluble proteins and grouped them by a hierarchical clustering based on the correlation coefficients of proteins, three distinct families of immune markers correlated positively with each other, although many of them showed broad positive correlation with outgroup members. Group I includes IL-4, IL-5, IL-13, TGF- $\beta$, CCL2 (MCP-1), CCL3 (MIP-1 $\alpha$ ), CCL5 (RANTES), CCL11 (Eotaxin-1), CXCL9 (MIG), C5a, and periostin; Group II contains IL-1 $\alpha$, IL-2, IL-7, IL-8, IL-15, TNF- $\alpha$, CCL4 (MIP-1 $\beta)$, CX3CL1 (Fractalkine), and calprotectin; Group III includes IL-6, IL-10, IL-21, IFN- $\gamma$, CXCL10 (IP-10), CXCL16, and ECP. It is notable that Group I included type 2 cytokines (IL-4, IL-5, IL-13, and TGF- $\beta$ ) and chemoattractants (CCL3, CCL5, CCL11, periostin, and C5a) for granulocytes, including eosinophils. ${ }^{16,21}$ On the other hand, Group II primarily include various cytokines linked to cytokine release syndrome (IL-1 $\alpha$, IL-6, IL-10, and TNF- $\alpha$ ), while Group III includes type 1 cytokines and chemokines involved in cell-mediated immunity (IL-2, IL-7, IL-15, IFN- $\gamma$, CXCL10, and CXCL16), indicating functional correlation of soluble mediators in inflamed respiratory environment during the acute phase of COVID-19. When we compared the overall levels of cytokines and chemokines between mild and severe groups, only three inflammatory markers, IL-6 (mean \pm S.D.: $2.8 \pm 8.1$ vs. $9.1 \pm 14.1 \mathrm{ng} / \mathrm{ml}$ for mild and severe group, respectively), TGF- $\beta(0.9 \pm 1.9$ vs. $3.2 \pm 4.0 \mathrm{ng} / \mathrm{ml}$ for mild and severe group, respectively), and ECP (as noted above), were significantly higher in severe patients than the mild group (Supplementary Table S3). Systemic elevation of IL-6 is known to be a hallmark of respiratory failure and cytokine release syndrome in severe COVID-19,,$^{22,23}$ and our data confirms a significant association of this inflammatory cytokine with disease severity even in the respiratory environment of COVID-19 patients. In addition, IL-6 also showed broad and 
224 significant association with pro-inflammatory cytokines, as well as type 2 cytokines. ECP is significantly correlated with proinflammatory cytokines, including IL-6 and TNF- $\alpha$, in addition to type 2 cytokines, such as IL-5 and IL-13 (Fig. 4a), suggesting a potential association of eosinophilic inflammation with cytokine release syndrome and type 2 immune responses. TGF$\beta$ was not significantly correlated with any other soluble markers, although it was grouped with type 2 cytokines. Given the functional role of TGF- $\beta$ in mucosal immunity and eosinophilic pneumonia, ${ }^{24,25}$ a significant elevation of TGF- $\beta$ in respiratory specimens from severe COVID19 patients can be indicative of eosinophilic and type 2 mucosal immune responses.

Since eosinophilic inflammation is associated with Th2-polarized immune responses in various pulmonary disorders, ${ }^{16,26}$ we next assessed viral antigen-specific antibody responses, with a focus on specific isotypes in respiratory secretions and plasma from the patients. We speculated that specific immune responses against the SARS-CoV-2 antigen are orchestrated by a Th2biased immune environment, which can drive preferential isotype switching of specific antibodies in B cells toward specific subclasses, such as $\operatorname{IgE} .{ }^{27}$ Antibody responses against SARS-CoV-2 N protein generally rose during the first 10 days after symptom onset, peaking at D10-D20, and then gradually declining in the respiratory specimens, regardless of disease severity (Fig. 5a). The levels of specific isotype responses against the viral antigen were not significantly different between mild and severe groups, except $\operatorname{IgG}$ which was significantly higher in severe cases, especially during D10-D20. The positive rate of anti-N-specific IgE response was $46.7 \%(7 / 15)$ in specimens from the severe group and $41.7 \%(5 / 12)$ in those from mild patients, indicating a general prevalence of $\operatorname{IgE}$ responses in respiratory tracts regardless of disease severity. Since there have been several reports on the involvement of elevated 
246 complement activation in the plasma of severe COVID-19 patients, ${ }^{28-30}$ we also assessed the 247 levels of complement protein in the respiratory specimens. We detected significantly higher 248 levels of $\mathrm{C} 3 \mathrm{a}$ with a peak response at around D10 in severe patients (Fig. 5b), indicating concomitant complement activation with antibody responses in the respiratory tracts during the acute phase of COVID-19. In order to examine antibody-dependent activation of the complement 251 pathway, ${ }^{30}$ we examined the correlation of $\mathrm{C} 3$ a concentration with anti-N $\operatorname{IgM}$ and $\operatorname{IgG}$ antibody responses, which are the major isotypes involved in the classical pathway of complement 253 activation. ${ }^{31}$ The concentration of C3a was significantly correlated with both IgM and IgG levels 254 when measured in the same respiratory specimen (Fig. 5c), suggesting activation of the classical 255 pathway elevated complement activation in the respiratory tract in severe COVID-19.

256 Next, we examined the systemic levels of antigen-specific antibody responses in plasma (Fig. 6a and Supplementary Fig. S7). Anti-N IgG antibodies, including IgG1 and IgG3 (IgG2 and IgG4 258 were barely detected), gradually increased in the plasma of all 15 patients (10 mild and 5 severe patients) examined. Specific IgA responses were detected in plasma from all the severe patients and positive in seven out of ten mild patients. In the case of $\operatorname{IgE}$, four out of five $(80 \%)$ severe cases showed detectable levels, whereas it was detectable in only two patients among ten (20\%) mild patients within 40 days after symptom onset (Supplementary Fig. S7). Antibody responses were generally more prompt and pronounced in severe cases than the mild group (Fig. 6a and Supplementary Fig. S7). Moreover, levels of all the antibody isotypes were significantly higher 265 in the severe group than mild patients. Notably, there was a consistent surge in specific $\operatorname{IgE}$ 266 levels against viral $\mathrm{N}$ protein with a peak response during D10-20 in all severe pneumonic 267 patients, except one (P13), indicating a prevalence of antigen-specific IgE responses in severe 268 patients. We assessed complement activation in the same set of plasma samples by measuring the 
concentrations of $\mathrm{C} 3 \mathrm{a}$ and $\mathrm{C} 5 \mathrm{a}$. As observed in respiratory specimens (Fig. 5b), levels of C3a and C5a were generally higher in plasma from severe patients $(7.9 \pm 1.3 \mu \mathrm{g} / \mathrm{ml}$ and $50.5 \pm 20.3 \mathrm{ng} / \mathrm{ml}$ for $\mathrm{C} 3 \mathrm{a}$ and $\mathrm{C} 5 \mathrm{a}$, respectively) than in mild group $(1.2 \pm 2.0 \mu \mathrm{g} / \mathrm{ml}$ and $38.9 \pm 30.1 \mathrm{ng} / \mathrm{ml}$ for C3a and C5a, respectively) (Fig. 6b). The difference in C5a levels between mild and severe groups was statistically significant, as previously reported. ${ }^{28}$ In addition, we observed a significant positive correlation of C5a levels with plasma IgG1 and IgG3 levels (Fig. 6c). These results confirm that viral antigen-specific antibody responses in plasma from severe patients is significantly higher than those from the mild group, as previously reported. ${ }^{32,33}$ This might be functionally linked to systemic and persistent activation of the complement cascade in severe pneumonic patients, potentially through the classical pathway. In addition, systemic but transient surge of antigen-specific IgE responses, driven by enhanced eosinophilic inflammation and TGF$\beta$ response in the respiratory tracts of severe patients, could be a hallmark of pathogenic progression and Th2-biased mucosal immunity in pneumonic lungs of severe COVID-19 patients. In order to confirm the pathogenic contribution of antibody responses and complement activation in severe COVID-19, we carefully assessed kinetic responses in the respiratory specimens and plasma from the fatal case, P15, and performed histological and immunofluorescence analysis using two lung biopsies obtained during late stage (D36 and D48) of COVID-19 before succumbing to death at D59 (Fig. 7). Viral loads in respiratory specimens gradually declined after symptom onset and were barely detected after D30 (Fig. 7a), as observed in other surviving cases (Fig. 1). Even though plasma CRP levels peaked at D13 and decreased thereafter, the levels of lactate dehydrogenase ( $\mathrm{LDH})$ remained above normal range throughout the disease course (Fig. 7b), suggesting persistent cellular damage. Histopathologic examination of lung biopsies 
291 from the deceased patient showed subpleural fibrosis and diffuse fibrin deposit at D36 after 292 symptom onset (Fig. 7c and d). Lung parenchyma was almost completely destroyed, evidenced 293 by the presence of interstitial thickening with fibrosis, smooth muscle proliferation, and granulation tissue formation. Alveolar spaces contained some macrophages and hemorrhage, some neutrophilic infiltration, and vascular congestion was observed in subpleural granulation tissue. At D48, hemorrhage and congestion were observed in destroyed lung parenchyma and focal pulmonary microthrombi were identified (Fig. 7d). We also observed parenchymal destruction by cystic change and bronchiolar metaplasia of alveoli. Infiltration of some lymphoplasma cells in fibrotic interstitium was noted and variable sized alveolar macrophages and seromucinous fluid were observed in alveolar spaces. Interestingly, antibody responses (IgM, $\mathrm{IgG}$, and $\mathrm{IgA}$ ) against viral $\mathrm{N}$ antigen in respiratory specimens peaked during D10-20, gradually declined until D30, and THEN elevated again thereafter (Fig. 7e). The kinetic responses of C5a in the respiratory samples were also biphasic, with second minor surge at D30-40, suggesting antibody-dependent complement activation in the respiratory tract. In addition, a transient surge of IgE response was observed right before the second rise of antibody and complement responses on D26. Moreover, N-specific antibody responses in plasma were also biphasic with an initial peak during D20-40 and a second rise after D50. Plasma C5a levels also showed a second surge after D50, indicating concomitant systemic responses of antibody and complement activation. These results suggest that a second round of antigenic stimulation may drive specific antibody responses and associated complement activation, especially in the respiratory tract. Since there was few viral release in respiratory specimens after D30, we suspected that there might be active production of viral antigens without secretion of virus particles, i.e. abortive viral infection, in inflamed lungs of COVID-19. Indeed, immunostaining of viral $\mathrm{N}$ antigen of lung biopsies 
314 collected at D36 and D48, active expression was especially pronounced in vascular walls, 315 including endothelial cells, as well as in a few epithelial cells (Fig. 7f). Detection of viral antigen 316 and/or viral RNA in endothelial cells and/or epithelial cells of tissue biopsies from COVID-19 317 patients has also been confirmed in other reports. ${ }^{19,34-39}$ In addition, formalin-fixed paraffinembedded lung tissue subjected to immunohistochemical staining with anti-C5b-9 antibody showed massive deposition of membrane attack complexes (MACs) in vascular walls, especially subendothelial and smooth muscle layers, bronchial epithelial cells, as well as inflammatory cells including macrophages and lymphocytes (Fig. 8a). The intensity of C5b-9 staining in pulmonary vessels was increased at D48 when compared to that of D38. The gradual increase of creatine phosphokinase (CPK) in the patient's plasma after D40 may also reflect progressive cellular damage of vascular smooth muscle layer upon MAC formation and/or myocardial injury in fatal COVID-19 patients (Fig. 7b), as previously reported. ${ }^{40,41}$ Moreover, deposition of IgG immune complexes in hyaline membranes and fibrin deposits within luminal spaces of airways and vascular capillaries emerged at D36 and increased at D48 (Fig. 8b and Supplementary Fig. S9). These results strongly suggest that active expression of viral antigen without producing viral particles during late stage of severe COVID-19 drives a second cycle of antibody responses accompanying complement activation. Concomitant immune complex formation and complement activation may drive irreversible pulmonary damage in fatal COVID-19. ${ }^{42}$ Evidence of fatal COVID-19 vasculopathy accompanying deposition of immune complexes and/or complement components inside vascular walls in multiple organs have also been reported in recent studies. ${ }^{43,44}$

Based on our observations of extensive kinetic analyses using respiratory specimens, we propose that sustained eosinophilic inflammation is followed by Th2-biased adaptive immune responses, 
337 as evidenced by enhanced viral antigen-specific antibody responses and concomitant 338 complement activation, in severe COVID-19. Overt antibody responses together with 339 complement activation potentially contribute to the progression and pathogenesis of severe 340 COVID-19. Nevertheless, significant infiltration of eosinophils into lungs of COVID-19 patients 341 during the acute phase of infection can be either protective or detrimental. ${ }^{45}$ Although peripheral 342 blood eosinopenia at initial presentation might be associated with severe COVID-19, as we and 343 others observed in COVID-19 patients (see complete blood count results in Supplementary Fig. 344 S8), ${ }^{46}$ sustained and enhanced eosinophilic inflammation in respiratory tracts of COVID-19 is 345 consistently observed in severe cases (Fig. 2 and 3b). Interestingly, rapid eosinophilic infiltration 346 into infected lungs was often observed in mild patients within 10 days after symptom onset, 347 whereas eosinophil infiltration is delayed but prolonged and eosinophilic inflammation is 348 significantly increased, as indicated by enhanced responses of ECP and EDN, in respiratory 349 tracts of severe pneumonic cases. Eosinophil response during the early stage of viral infection 350 can orchestrate antiviral responses to respiratory viruses by producing reactive oxygen species 351 and eosinophil-derived RNases (EDN/RNase2 and ECP/RNase3). ${ }^{45}$ However, sustained 352 eosinophilic inflammation may represent an acute type I hypersensitivity reaction, which is often 353 functionally linked to Th2-polarizing respiratory environment. ${ }^{26}$ Enhanced TGF- $\beta$ responses, 354 mast cell activation, and more prompt and robust viral antigen-specific antibody responses, 355 including IgE isotypes, strongly support the potential pathogenic role of eosinophilic 356 inflammation and Th2-biased mucosal immunity in severe COVID-19. ${ }^{6}$ Moreover, acute and 357 transient surge of eosinophilia in inflamed lungs, as observed in the fatal case (P15), might be a 358 critical driver of irreversible lung damage associated with lethal outcome in severe COVID-19 359 patients. Recently, the association of systemic eosinophilic inflammation with various COVID- 
19-associated syndromes has been reported by several studies. ${ }^{47,48}$ Interestingly, we observed transient elevation, peaking at D10, of macrophage activation as assessed by sCD163 levels in respiratory samples of severe patients (Fig. 3d). The role of alternatively activated (M2 type) macrophages in eosinophilic pulmonary inflammation has also been previously documented. ${ }^{16,49}$ Therefore, macrophage activation syndrome often observed in severe COVID-19 patients ${ }^{17}$ can be functionally linked to eosinophilic pulmonary inflammation. While the causal relationship and precise mechanisms mediating innate cellular interactions during pathologic progression remain to be elucidated, our kinetic analyses reveal concomitant elevation of pulmonary inflammation, potentially driven by macrophage, eosinophil, and mast cell activation in severe COVID-19 (Fig. 3). Given that early viral loads in respiratory tracts of COVID-19 patients does not seem to enhance pulmonary inflammation by innate cellular activation, host factor(s) specifically driving inflammation need to be determined in future studies.

Our second critical finding is the potential role of enhanced antibody responses accompanying elevated complement activation in disease pathogenesis of severe COVID-19. As mentioned above, kinetic analyses of respiratory specimens revealed sustained and higher eosinophilic inflammation as a potential driver for Th2 immune responses in severe COVID-19. A recent immune profiling study using peripheral blood mononuclear cells and plasma samples from 113 patients with moderate or severe COVID-19 also showed an association of an increase in multiple type 2 effectors, including IL-5, IL-13, IgE, and eosinophils, with disease severity. ${ }^{6}$ More rapid and robust antibody responses specific to SARS-CoV-2 antigens, including spike and nucleocapsid $(\mathrm{N})$, have been consistently observed in many other studies. ${ }^{32,33,50}$ Here, we confirmed enhanced antibody responses against viral $\mathrm{N}$ antigen in plasma, as well as respiratory IgG response, in severe patients. In addition, kinetic analysis revealed earlyer peak response 
383 (D10-20) of systemic IgA and IgE, as well as respiratory antibodies, followed by systemic IgG 384 responses which peak at D20-30 in severe cases; meanwhile, all these isotypes peaked at D20-30 385 in plasma from mild group patients (Fig. 5). Earlier predominance of $\operatorname{IgA}$ and $\operatorname{IgE}$ responses might be due to pulmonary eosinophilic inflammation and enhanced TGF- $\beta$ responses in 387 respiratory mucosal environment of severe patients. ${ }^{16,24,26}$ Moreover, complement activation was generally higher in the respiratory tracts and systemic circulation of severe patients than mild 389 group, and correlated well with the levels of $\operatorname{IgM}$ and $\operatorname{IgG}$ responses in the respiratory environment, suggesting increased activation of antibody-dependent classical pathway. While the contribution of both alternative and lectin-dependent pathways during SARS-CoV-2 infection can not be excluded, ${ }^{44}$ complement $\mathrm{Clq}$ levels in plasma from severe cases were lower than mild cases ${ }^{30}$ indicating systemic $\mathrm{Clq}$ depletion due to antibody-dependent classical pathway of 394 complement activation in severe cases. Nevertheless, it remains unknown whether enhanced antibody responses and accompanying complement activation are directly and functionally associated with pathogenic progression of severe COVID-19. ${ }^{51,52}$ Enhanced antibody responses are not necessarily critical for antiviral immunity, since viral copy numbers in respiratory secretions declined to baseline during peak antibody responses (D20-30) and there was no significant difference in viral loads between mild and severe patients (Fig. 1a). In addition, 400 systemic viremia has been rarely detected in the majority of COVID-19 patients regardless of 401 disease severity. ${ }^{53,54}$ Rather, we suspected that Fc $\gamma$ receptor (Fc $\left.\gamma \mathrm{R}\right)$-mediated inflammation upon 402 crosslinking by immune complexes induces lung tissue damage through activation of the 403 complement pathway and MAC formation (Fig 8). ${ }^{52}$ Since viral release from infected host cells 404 rapidly decreased, rising antibodies might bind to cells expressing the viral antigens. Infected 
405

406

407

408

409

410

411

412

413

414

415

416

417

418

419

420

421

422

423

424

425

426

427

epithelial and endothelial cells (Fig. 7f) in lungs of COVID-19 patients could be primary targets for the antigen-antibody immune complexes. Gradually increased IgG deposition on the luminal surfaces of airways and vasculatures observed in lung biopsies from a fatal case (Fig. 8b) strongly supports the pathogenic role of the immune complex-mediated pathway. We also often observed large immune complexes including detached epithelial layers or $\mathrm{N}$ antigen-positive cells in airway lumens (Fig 8b and Supplementary Fig. S9). Expression of viral antigens in epithelial and endothelial cells without producing viral particles, indicating abortive viral infection, during late stage COVID-19 may further fuel antibody responses and aggravate immune-complex-mediated inflammation, including complement activation (Fig 7d and Fig. 8), as well as activating effector leukocytes by engaging various members of Fc $\gamma \mathrm{R} .{ }^{52}$ To confirm this, we retrieved gene expression data sets from recent single cell RNA sequencing (scRNA seq) studies ${ }^{55,56}$ and analyzed the hallmark gene set scores for each signaling pathway. ${ }^{57}$ In the initial analyses of signature gene set scores for inflammation in major leukocyte populations (Fig. 9a), myeloid cells including monocytes, macrophages, and dendritic cells, as well as neutrophils showed prominent increase in hallmark inflammation scores when compared to those of healthy control group (Fig. 9b). Analysis of scRNA seq data sets from mononuclear phagocytes (monocytes, macrophages, and dendritic cells) and neutrophils showed significantly higher levels of hallmark gene set scores for both Fc $\gamma \mathrm{R}$ signaling and complement activation in severe group than those of mild cases (Fig. 9c). These results clearly indicate a significant role of Fc $\gamma \mathrm{R}$ signaling and complement activation in inflammatory responses and pathogenic contributions by phagocytic cells. ${ }^{17,29,52}$

In conclusion, our current kinetic analyses of respiratory specimens from COVID-19 patients clearly reveal an association of eosinophilic pulmonary inflammation with severe pneumonic 
428 pathogenesis. These observations require further confirmation with extensive research of the 429 pathogenesis of eosinophilic inflammation and its association with pathogenic role of antibody 430 responses and complement activation in order to establish proper therapeutic strategies against 431 severe pneumonia often resulting in fatal outcome in COVID-19. Moreover, potential abortive 432 viral infections during late stage of COVID-19 may need to be carefully controlled to abrogate 433 the pathologic contribution by immune-complex and complement-mediated inflammation.

\section{Methods}

\section{Ethnical statement}

437 The current research was approved by the institutional review boards of Chosun University 438 Hospital (IRB no.: 2020-02-011), Chungnam National University Hospital (IRB no.: 439 CNUH2017-12-004), and Seoul National University Hospital (IRB no.: C-1509-103-705). This 440 study was performed in accordance with the ethical standards laid down in the 1964 declaration 441 of Helsinki and all subsequent revisions. This study was conducted with informed consent from 442 patients or their legal guardians.

\section{Patient groups}

444 General information on the clinical courses and baseline characteristics of the COVID-19 445 patients included in this study are summarized in Supplementary Table S1 and Fig. S1. The 446 patients were divided into two groups based on disease severity. The mild group includes 36 447 patients who were asymptomatic, with mild respiratory symptoms but no detectable pneumonia, 448 or with mild to moderate pneumonia determined by chest imaging and clinical symptoms. The 449 severe group includes 16 patients who suffered from severe pneumonia requiring high flow 
464 microscope.

\section{Flow cytometry}

\section{Cytological analysis}

oxygen supply and/or mechanical ventilation. Among the severe group patients, all the patients survived and were discharged, except one patient (P15) who succumbed to death due to fatal ARDS. The patients were also divided into two sets. Group 1 includes 15 patients (10 mild and 5 severe group patients) who provided blood and respiratory specimens at different time points after symptoms onset. Group 2 includes 37 patients (26 mild and 11 severe patients) and provided respiratory specimens during the acute phase of COVID-19. Some of the clinical data from three patients (ID: 1, 11, and 12) were included in our previous preprint paper (DOI:10.21203/ rs.3.rs-23607/v1).

BALFs and sputa samples collected from the patients were smeared on glass slides immediately after collection. The slides were then immediately fixed with $95 \%$ ethanol in a Coplin jar. After fixing the slides, hematoxylin and eosin (H\&E) staining was performed. The presence and relative proportions of various leukocyte subsets were estimated under light microscope independently by two experienced pathologists and consensus was reached under multi-head

Peripheral blood leukocytes and mononuclear cells (PBMCs) were prepared by standard density gradient centrifugation with Histopaque-1077 and 1119 (Sigma-Aldrich, St. Louis, MO, USA) in a biosafety level 3 laboratory in Seoul National University. Isolated blood leukocytes were directly stained with antibodies listed below for flow cytometry in a biosafety level 3 laboratory. Cells in BALFs and lung biopsies were directly prepared and analyzed on the collection day in a biosafety level 3 laboratory, as previously described. ${ }^{58}$ Dead cells were stained with Zombie Aqua Fixable Viability Dye (BioLegend, San Diego, CA, USA). Cells were stained with the 
473 following sets of antibodies differentially labeled with the indicated fluorochrome; anti-CD4474 Alexa488, anti-CD8-PerCP or APC, anti-CD14-BV605, anti-CD16-Alexa700, anti-CD24-

475 BV421, anti-CD45-PerCP-Cy5.5, anti-CD206-Alexa488, anti-HLA-DR-BV711 (from 476 BioLgend), anti-CD3-PE-CF594 or Pacific blue, anti-CD20-APC/H7, and anti-CD56-APC (from BD Bioscience). Cell were then fixed with a fixation buffer (BD Bioscience) and analyzed using 478 a FACS Fortessa II flow cytometer (BD Biosciences). Data were analyzed using Flowjo software 479 (Tree Star, Ashland, OR, USA). Gating strategies for flow cytometric analyses are summarized in $480 \quad$ Supplementary Fig. S2.

\section{Quantitation of cytokines and chemokines}

482 Cytokine/chemokine levels in human respiratory samples (BALFs and sputa) were measured by 483 a luminex multiplex assay system (Luminex, Austin, TX, USA) after gamma-irradiation (30 kGy). Luminx assay was run according to the manufacturer's instructions, using a customized

485 human cytokine multiplex panel (R\&D Systems, Inc. Minneapolls, MN, USA). The panel 486 included: CCL2/JE/MCP-1, CCL3/MIP-1 $\alpha$, CCL4/MIP-1 $\beta$, CCL5/RNATES, CCL11/Eotaxin, 487 complement component C5a, CX3CL1/Fractalkine, CXCL9/MIG, CXCL10/IP-10/CRG-2, 488 CXCL16, IFN- $\gamma$, IL-1 $\alpha /$ IL-1F1, IL-2, IL-4, IL-5, IL-6, IL-7, IL-8/CXCL8, IL-10, IL-12/IL489 23p40, IL-13, IL-15, IL-21, Periosin/OSF-2, TNF- $\alpha$, TGF- $\beta$, sCD163, and granzyme A. Assay 490 plates were measured using a Luminex 100/200TM analyzer (Luminex, Austin, TX, USA). 491 Standard curve for each cytokine/chemokine was drawn using the supplied cytokine/chemokine 492 standard and determined with the best fit algorithm using MasterPlex QT 2010 software 493 (MiraiBio, Hitachi, CA, USA). IL-33 (Invitrogen, Carlsbad, CA, USA), lipocalin-2 (Abcam, 494 Cambridge, UK), EDN, and MCT (Cusabio, Houston, TX, USA) were quantified using human 
495

496

497

498

499

500

501

502

503

504

505

506

507

508

509

510

511

512

513

514

515

516

517

ELISA kits according to the manufacturer's instruction. Concentration of ECP and calprotectin in respiratory specimens were measured via clinical diagnosis service from Seoul Clinical Laboratory (Seoul, Republic of Korea).

\section{Enzyme-linked immunosorbent assay (ELISA)}

To assess for SARS-CoV-2 N protein-specific antibody responses, 96-well immunoassay plates (Nunc, Waltham, MA, USA) were coated with $100 \mu \mathrm{L}$ of purified antigen at a concentration of 1 $\mu \mathrm{g} / \mathrm{mL}$ at $4{ }^{\circ} \mathrm{C}$ overnight. The plates were then blocked for $2 \mathrm{~h}$ at room temperature (RT) with PBS containing 5\% skim milk. One hundred microliters of serially diluted plasma or respiratory samples were incubated for $2 \mathrm{~h}$ at RT. After washing with PBS containing $0.05 \%$ Tween20 (0.05\% PBST), horseradish peroxidase (HRP)-conjugated mouse anti-human IgG1, IgG2, IgG3, IgG4, IgG, IgM, IgA or IgE antibody (Southern Biotech, Birmingham, AL, USA) was added and incubated for $1 \mathrm{~h}$ at RT. Wells were washed with $0.05 \%$ PBST and incubated with a 3,3',5,5'tetramethylbenzidine (TMB) peroxidase substrate solution (KPL, Gaithersburg, MD, USA) for 10min. The reactions were stopped by addition of a $1 \mathrm{M}$ phosphoric acid solution. Absorbance was measured at $450 \mathrm{~nm}$ using a microplate reader (Beckman Coulter, Brea, CA, USA). The cutoff titer for the ELISA was defined as the lowest titer showing an optical density (OD) over the mean OD plus $3 \times$ standard deviation (s.d.) from three control plasma samples (diluted 1:10) collected from healthy volunteers or three pneumonia patients who were never diagnosed with COVID-19.

\section{Quantitation of viral loads}

Real-time reverse transcription-polymerase chain reaction (RT-PCR) assay for detection of SARS-CoV-2 was performed according to the manufacturer's instructions (Kogenebiotech, Seoul, South Korea). Total RNAs were obtained from nasopharyngeal and throat swab samples (upper 
respiratory tract) and sputa (lower respiratory tract). Primer sets targeting E and RdRP genes of SARS-CoV-2 were used with a cut-off cycle threshold $(\mathrm{Ct})$ value of higher than 38 cycles.

\section{Quantitation of complements}

Human complement assays were conducted using quantitative C3a and C5a ELISA kits according to the manufacturer's instructions (Thermo Fisher Scientific, Waltham, MA, USA). In brief, patients' plasma were incubated in microwells adsorbed with anti-human C3a or C5a coating antibody for $2 \mathrm{~h}$ at RT, washed 6 times, and incubated with a biotin-conjugated antihuman $\mathrm{C} 3 \mathrm{a}$ or $\mathrm{C} 5 \mathrm{a}$ antibody for $1 \mathrm{~h}$. The plates were then washed 6 times followed by incubation with streptavidin-HRP for $1 \mathrm{~h}$. After washing the wells, TMB substrate solution was added and further incubated at RT. The reaction was terminated by adding $1 \mathrm{M}$ phosphoric acid and absorbance was measured with a TECAN microplate reader (TECAN, Mannedorf, Switzerland) at $450 \mathrm{~nm}$.

\section{Immunohistochemistry and immunofluorescence assay using lung biopsies}

Histopathological analysis of lung biopsies was performed after fixation in $10 \%$ formalin and embossing in paraffin. Tissue sections ( $4 \mu \mathrm{m}$ thickness) were stained with hematoxylin and eosin (H\&E) and two experienced pathologists specialized in lung pathology evaluated the H\&E slides under a light microscope (Olympus BX-53; Olympus, Tokyo, Japan). For immunohistochemistry and immunofluorescence analysis, paraffin-embedded tissue sections were placed at $55^{\circ} \mathrm{C}$ overnight. Tissue sections were rehydrated by submerging in xylen sequentially for 15 min, 5 min, and 5 min, followed by immersion in $100 \%, 100 \%, 95 \%, 90 \%, 80 \%$, and $70 \%$ ethanol in serial order, for 3 min per step. For antigen retrieval, the slides were soaked and heated in HIER buffer (10 mM Tri-sodium citrate, $0.05 \%$ Tween-20, $\mathrm{pH} \mathrm{6)}$ in the microoven for $20 \mathrm{~min}$. After blocking with a buffer containing 5\% BSA and Fc blocker (BD Biosciences) for $1 \mathrm{~h}$ at room 
541 temperature, autofluorescence emitted by lung tissues was inhibited by incubation with $0.1 \%$

542 Sudan Black solution for $20 \mathrm{~min}$, followed by incubation with indicated antibody for 15 minutes.

543 The antibodies used to detect specific antigens for immunostaining processes were rabbit 544 polyclonal anti-SARA-CoV-2 N antibody was obtained from Sino biological (Beijing, China)

545 and anti-CD31 and pan-cytokeratin (CK) antibodies purchased from DAKO (Carpinteria, CA, 546 USA). Antibody binding to the cells in sections was detected using horseradish peroxidase 547 (HRP) reaction kits (DAKO) for immunohistochemistry or Alexa488-, Alexa594-, Alexa-633 548 conjugated secondary antibodies (Molecular Probes) for immunofluorescence. Nuclear DNA was 549 counterstained with 4,6-diamidino-2-phenylindole (DAPI). Confocal microscopy was performed 550 using an OlympusFV3000 laser-scanning microscope (Olympus). All images were analyzed and 551 processed using the Olympus Fluoview or Adobe Photoshop software. Analysis of single cell RNA seq datasets of BALFs from COVID-19 patients.

553 We collected BALF single cell RNA seq (scRNA-seq) datasets from the Gene Expression 554 Omnibus (GEO) under accession code GSE145926 and from FigShare 555 (https://doi.org/10.6084/m9.figshare.12436517). ${ }^{55,56}$ We applied quality control criteria to each 556 data set (mitochondrial gene percentage $<0.15$ ). After filtering, a total single cell of 182,140 557 were used for analysis. To remove batch effects across the two datasets, we used multi canonical 558 correlation analysis 3 (CCA3) in Seurat3 R package. ${ }^{59}$ Variably expressed genes were selected using the FindVariableGenes function in default parameters of Seurat v3.1.5. ${ }^{59}$ Cell clustering 560 and Uniform Manifold Approximation and Projection (UMAP) visualization were performed 561 using the FindClusters and RunUMAP functions, respectively. The annotations of cell identity 562 for each cluster were defined by the expression of known marker genes: epithelial cells, EFHC1 563 and MLF1; T/NK cells, IL32, KLRB1, and STMN1; B cell, CD19 and MS4A1; neutrophils, 
564 LYN, FCGR3B, and ITGAX; monocyte/macrophage/dendritic cells, CD14, CCL2, CD68, 565 FABP5, CD74, CLEC4C, KIT, CPA3, HPGD, and LTC4S. To score the hallmark gene sets for 566 inflammation, ${ }^{60}$ complement activation $^{60}$, and reactome $\mathrm{Fc} \gamma \mathrm{R}$ activation, ${ }^{61}$ data sets were 567 downloaded from MsigDB (https://www.gsea-msigdb.org/gsea/msigdb) using the 568 AddModuleScore function provided by the Seurat package and analyzed.

\section{Statistical analysis}

570 Data was analyzed using the Graph Pad Prism 5.01 software (GraphPad Software, La Jolla, CA, 571 USA) and Microsoft Excel (Microsoft Office Professional Plus 2016). Statistical analyses were 572 performed using a two-tailed Mann-Whitney U test, unpaired Student's $t$-test, or one-way 573 analysis of variance (ANOVA) followed by Newman-Keuls t-test for comparisons of values 574 among different groups. Spearman's rank test was used to analyze the correlation between 575 variables. A $p$-value of $<0.05$ was considered statistically significant.

\section{Acknowledgement}

This research was supported by a grant from the National Research Foundation of Korea (grant no. 2017M3A9E4061998), and a grant (2017N-ER5307/4834-300-210-13) from Korea Center for Disease Control and Prevention, funded by the government of South Korea. Y.K., U.P.,

581 N.Y.H., H.P., K.J., and J.G.K. received a scholarship from the BK21-plus education program 582 provided by the National Research Foundation of Korea.

Potential conflicts of interest. All authors: No reported conflicts of interest. 
586

5871 Grp, C. S. The species Severe acute respiratory syndrome-related coronavirus: classifying

588

589

590

591

592

593

594

595

596

5974

598

599

$600 \quad 5$

601

602

$603 \quad 6$

604

605

606

607 2019-nCoV and naming it SARS-CoV-2. Nature Microbiology 5, 536-544, doi:10.1038/s41564-020-0695-z (2020).

2 Yang, X. et al. Clinical course and outcomes of critically ill patients with SARS-CoV-2 pneumonia in Wuhan, China: a single-centered, retrospective, observational study. Lancet Respir Med, doi:10.1016/S2213-2600(20)30079-5 (2020).

3 Kim, E. S. et al. Clinical Course and Outcomes of Patients with Severe Acute Respiratory Syndrome Coronavirus 2 Infection: a Preliminary Report of the First 28 Patients from the Korean Cohort Study on COVID-19. J Korean Med Sci 35, e142, doi:10.3346/jkms.2020.35.e142 (2020).

Zhou, F. et al. Clinical course and risk factors for mortality of adult inpatients with COVID-19 in Wuhan, China: a retrospective cohort study. Lancet 395, 1054-1062, doi:10.1016/S0140-6736(20)30566-3 (2020).

5 Tay, M. Z., Poh, C. M., Renia, L., MacAry, P. A. \& Ng, L. F. P. The trinity of COVID-19: immunity, inflammation and intervention. Nat Rev Immunol, doi:10.1038/s41577-0200311-8 (2020).

6 Lucas, C. et al. Longitudinal analyses reveal immunological misfiring in severe COVID19. Nature 584, 463-469, doi:10.1038/s41586-020-2588-y (2020).

7 Mathew, D. et al. Deep immune profiling of COVID-19 patients reveals distinct immunotypes with therapeutic implications. Science 369, doi:10.1126/science.abc8511 (2020). 
$6088 \mathrm{Li}, \mathrm{H}$. et al. SAA is a biomarker to distinguish the severity and prognosis of Coronavirus 609 Disease 2019 (COVID-19). J Infect, doi:10.1016/j.jinf.2020.03.035 (2020).

6109 Pan, Y., Zhang, D., Yang, P., Poon, L. L. M. \& Wang, Q. Viral load of SARS-CoV-2 in 611 clinical samples. Lancet Infect Dis 20, 411-412, doi:10.1016/S1473-3099(20)30113-4 (2020).

61310 He, X. et al. Temporal dynamics in viral shedding and transmissibility of COVID-19. Nature medicine 26, 672-675, doi:10.1038/s41591-020-0869-5 (2020).

61511 Peng, L. S. et al. Altered phenotypic and functional characteristics of CD3+CD56+ NKT616 like cells in human gastric cancer. Oncotarget 7, 55222-55230, 617 61812 Yu, Y. R. et al. Flow Cytometric Analysis of Myeloid Cells in Human Blood, 619 Bronchoalveolar Lavage, and Lung Tissues. Am J Respir Cell Mol Biol 54, 13-24, 620 doi:10.1165/rcmb.2015-0146OC (2016).

62113 Hara, A. et al. S100A9 in BALF is a candidate biomarker of idiopathic pulmonary 622 62314 Pechous, R. D. With Friends Like These: The Complex Role of Neutrophils in the 624 625 62615 Spencer, L. A., Bonjour, K., Melo, R. C. \& Weller, P. F. Eosinophil secretion of granule627 derived cytokines. Frontiers in immunology 5, 496, doi:10.3389/fimmu.2014.00496 628 (2014).

62916 Rosenberg, H. F., Dyer, K. D. \& Foster, P. S. Eosinophils: changing perspectives in health 
and disease. Nat Rev Immunol 13, 9-22, doi:10.1038/nri3341 (2013).

17 Merad, M. \& Martin, J. C. Pathological inflammation in patients with COVID-19: a key role for monocytes and macrophages. Nat Rev Immunol 20, 355-362, doi:10.1038/s41577-020-0331-4 (2020).

18 Shoenfeld, Y. Corona (COVID-19) time musings: Our involvement in COVID-19 pathogenesis, diagnosis, treatment and vaccine planning. Autoimmunity Reviews 19, doi:ARTN 102538 10.1016/j.autrev.2020.102538 (2020).

19 Zeng, Z. et al. Pulmonary Pathology of Early Phase COVID-19 Pneumonia in a Patient with a Benign Lung Lesion. Histopathology, doi:10.1111/his.14138 (2020).

20 Reber, L. L., Hernandez, J. D. \& Galli, S. J. The pathophysiology of anaphylaxis. $J$ Allergy Clin Immunol 140, 335-348, doi:10.1016/j.jaci.2017.06.003 (2017).

21 Blanchard, C. et al. Periostin facilitates eosinophil tissue infiltration in allergic lung and esophageal responses. Mucosal Immunol 1, 289-296, doi:10.1038/mi.2008.15 (2008).

22 Mazzoni, A. et al. Impaired immune cell cytotoxicity in severe COVID-19 is IL-6 dependent. J Clin Invest, doi:10.1172/JCI138554 (2020).

23 Gubernatorova, E. O., Gorshkova, E. A., Polinova, A. I. \& Drutskaya, M. S. IL-6: Relevance for immunopathology of SARS-CoV-2. Cytokine Growth Factor Rev 53, 1324, doi:10.1016/j.cytogfr.2020.05.009 (2020).

24 Borsutzky, S., Cazac, B. B., Roes, J. \& Guzman, C. A. TGF-beta receptor signaling is critical for mucosal IgA responses. Journal of Immunology 173, 3305-3309, doi:DOI 10.4049/jimmunol.173.5.3305 (2004).

25 Katoh, S. et al. Elevated levels of periostin and TGF-betal in the bronchoalveolar lavage 
652

653

654

655

656

657

658

659

660

661

662

663

664

665

666

667

668

669

670

671

672

673

674

fluid of patients with idiopathic eosinophilic pneumonia. Asian Pac J Allergy Immunol, doi:10.12932/AP-111018-0414 (2019).

26 De Giacomi, F., Vassallo, R., Yi, E. S. \& Ryu, J. H. Acute Eosinophilic Pneumonia. Causes, Diagnosis, and Management. Am J Respir Crit Care Med 197, 728-736, doi:10.1164/rccm.201710-1967CI (2018).

27 Scott-Taylor, T. H., Axinia, S. C., Amin, S. \& Pettengell, R. Immunoglobulin G; structure and functional implications of different subclass modifications in initiation and resolution of allergy. Immun Inflamm Dis 6, 13-33, doi:10.1002/iid3.192 (2018).

28 Carvelli, J. et al. Association of COVID-19 inflammation with activation of the C5aC5aR1 axis. Nature, doi:10.1038/s41586-020-2600-6 (2020).

29 Cugno, M. et al. Complement activation in patients with COVID-19: A novel therapeutic target. J Allergy Clin Immunol 146, 215-217, doi:10.1016/j.jaci.2020.05.006 (2020).

$30 \mathrm{Wu}, \mathrm{Y}$. et al. Clinical Characteristics and Immune Injury Mechanisms in 71 Patients with COVID-19. mSphere 5, doi:10.1128/mSphere.00362-20 (2020).

31 Ricklin, D., Reis, E. S. \& Lambris, J. D. Complement in disease: a defence system turning offensive. Nat Rev Nephrol 12, 383-401, doi:10.1038/nrneph.2016.70 (2016).

32 Long, Q. X. et al. Antibody responses to SARS-CoV-2 in patients with COVID-19. Nature medicine 26, 845-848, doi:10.1038/s41591-020-0897-1 (2020).

33 Wang, Y. et al. Kinetics of viral load and antibody response in relation to COVID-19 severity. J Clin Invest, doi:10.1172/JCI138759 (2020).

34 Colmenero, I. et al. SARS-CoV-2 endothelial infection causes COVID-19 chilblains: histopathological, immunohistochemical and ultrastructural study of seven paediatric cases. Br J Dermatol, doi:10.1111/bjd.19327 (2020). 
67535 Varga, Z. et al. Endothelial cell infection and endotheliitis in COVID-19. Lancet 395,

676

$67736 \quad$ Hanley, B. et al. Histopathological findings and viral tropism in UK patients with severe 678

679

680

681

682

683

684

685

686

687

688

689

$690 \quad 41$

691

692

693

694

695

696

1417-1418, doi:10.1016/S0140-6736(20)30937-5 (2020).

fatal COVID-19: a post-mortem study. Lancet Microbe, doi:10.1016/S26665247(20)30115-4 (2020).

37 Borczuk, A. C. et al. COVID-19 pulmonary pathology: a multi-institutional autopsy cohort from Italy and New York City. Mod Pathol, doi:10.1038/s41379-020-00661-1 (2020).

38 Yao, X. H. et al. Pathological evidence for residual SARS-CoV-2 in pulmonary tissues of a ready-for-discharge patient. Cell Res 30, 541-543, doi:10.1038/s41422-020-0318-5 (2020).

39 Ackermann, M. et al. Pulmonary Vascular Endothelialitis, Thrombosis, and Angiogenesis in Covid-19. N Engl J Med 383, 120-128, doi:10.1056/NEJMoa2015432 (2020).

40 Clark, J. F. The creatine kinase system in smooth muscle. Mol Cell Biochem 133-134, 221-232, doi:10.1007/BF01267956 (1994).

41 Shi, L., Wang, Y., Wang, Y., Duan, G. \& Yang, H. Meta-Analysis of Relation of Creatine kinase-MB to Risk of Mortality in Coronavirus Disease 2019 Patients. Am J Cardiol 130, 163-165, doi:10.1016/j.amjcard.2020.06.004 (2020).

42 Ward, P. A., Fattahi, F. \& Bosmann, M. New Insights into Molecular Mechanisms of Immune Complex-Induced Injury in Lung. Frontiers in immunology 7, doi:ARTN 86 10.3389/fimmu.2016.00086 (2016).

43 Roncati, L. et al. Type 3 hypersensitivity in COVID-19 vasculitis. Clin Immunol 217, 
108487, doi:10.1016/j.clim.2020.108487 (2020).

44 Magro, C. et al. Complement associated microvascular injury and thrombosis in the pathogenesis of severe COVID-19 infection: A report of five cases. Transl Res 220, 1-13, doi:10.1016/j.trs1.2020.04.007 (2020).

45 Lindsley, A. W., Schwartz, J. T. \& Rothenberg, M. E. Eosinophil responses during COVID-19 infections and coronavirus vaccination. J Allergy Clin Immunol 146, 1-7, doi:10.1016/j.jaci.2020.04.021 (2020).

46 Qin, C. et al. Dysregulation of immune response in patients with COVID-19 in Wuhan, China. Clin Infect Dis, doi:10.1093/cid/ciaa248 (2020).

47 Leis-Dosil, V. M., Saenz Vicente, A. \& Lorido-Cortes, M. M. Eosinophilic Panniculitis Associated With COVID-19. Actas Dermosifiliogr, doi:10.1016/j.ad.2020.05.003 (2020).

48 Craver, R. et al. Fatal Eosinophilic Myocarditis in a Healthy 17-Year-Old Male with Severe Acute Respiratory Syndrome Coronavirus 2 (SARS-CoV-2c). Fetal Pediatr Pathol, 1-6, doi:10.1080/15513815.2020.1761491 (2020).

49 Dasgupta, P. \& Keegan, A. D. Contribution of alternatively activated macrophages to allergic lung inflammation: a tale of mice and men. $J$ Innate Immun 4, 478-488, doi:10.1159/000336025 (2012).

50 Atyeo, C. et al. Distinct Early Serological Signatures Track with SARS-CoV-2 Survival. Immunity, doi:10.1016/j.immuni.2020.07.020 (2020).

51 Zohar, T. \& Alter, G. Dissecting antibody-mediated protection against SARS-CoV-2. Nat Rev Immunol 20, 392-394, doi:10.1038/s41577-020-0359-5 (2020).

52 Bournazos, S., Gupta, A. \& Ravetch, J. V. The role of IgG Fc receptors in antibodydependent enhancement. Nat Rev Immunol, doi:10.1038/s41577-020-00410-0 (2020). 
72053 Chang, L., Yan, Y. \& Wang, L. Coronavirus Disease 2019: Coronaviruses and Blood Safety. Transfus Med Rev 34, 75-80, doi:10.1016/j.tmrv.2020.02.003 (2020).

72254 Huang, C. et al. Clinical features of patients infected with 2019 novel coronavirus in Wuhan, China. Lancet 395, 497-506, doi:10.1016/S0140-6736(20)30183-5 (2020).

55 Chua, R. L. et al. COVID-19 severity correlates with airway epithelium-immune cell interactions identified by single-cell analysis. Nat Biotechnol 38, 970-979, doi:10.1038/s41587-020-0602-4 (2020).

72756 Liao, M. et al. Single-cell landscape of bronchoalveolar immune cells in patients with COVID-19. Nature medicine 26, 842-844, doi:10.1038/s41591-020-0901-9 (2020).

72957 Pont, F., Tosolini, M. \& Fournie, J. J. Single-Cell Signature Explorer for comprehensive 730 visualization of single cell signatures across scRNA-seq datasets. Nucleic Acids Res 47, e133, doi:10.1093/nar/gkz601 (2019).

73258 Baharom, F. et al. Human Lung Dendritic Cells: Spatial Distribution and Phenotypic 733

59 Stuart, T. et al. Comprehensive Integration of Single-Cell Data. Cell 177, 1888-1902 e1821, doi:10.1016/j.cell.2019.05.031 (2019).

73760 Liberzon, A. et al. The Molecular Signatures Database (MSigDB) hallmark gene set 738 collection. Cell Syst 1, 417-425, doi:10.1016/j.cels.2015.12.004 (2015). doi:10.1093/nar/gkz1031 (2020). 


\section{Figure legends}

Fig. 1. Kinetic changes of respiratory viral loads and $\mathrm{C}$-reactive proteins in plasma. a. Kinetic changes of viral loads were evaluated in respiratory specimens (nasopharygeal/throat swabs for upper respiratory tract and sputa for lower respiratory tract) by quantitative RT-PCR targeting the E gene of SARS-CoV-2 and presented as Ct values. Blue circles $(n=49$ and 41 for upper and lower respiratory specimens, respectively) are from eight mild patients and red circles ( $n=38$ and 39 for upper and lower respiratory specimens, respectively) are from five severe cases. The blue and red lines show the trend in viral load, using smoothing splines (dashed line: cut-off value). Distribution of $\mathrm{Ct}$ values in mild (M) and severe (S) are also presented as violin plots (right graph, black line: median). b. Kinetic change of C-reactive proteins (CRP) in plasma is presented. Blue circles are from nine mild patients $(n=37)$ and red circles are from five severe cases $(n=23)$. Distribution of CRP levels in mild $(\mathrm{M})$ and severe $(\mathrm{S})$ are also presented as violin plots (right graph, black line: median). DPS: days post symptom onset. $* * *: p<0.001$ (Twotailed Mann-Whitney test).

\section{Fig. 2. Kinetic changes in respiratory specimens collected from COVID-19 patients. a.} Representative images of cytological analysis of sputa and BALFs by hematoxylin and eosin (H\&E) staining. The patient's ID., collection day after symptom onset, and types of specimens (SPT: sputum, and BALF: bronchoalveolar lavage fluid) are presented in the upper left text box. Arrows indicate PMNs (orange, mostly neutrophils), eosinophils (red), lymphocytes (blue), and macrophages (green). Scale bar: $20 \mu \mathrm{m}$. b. Relative frequencies of the indicated leukocyte subsets among $\mathrm{CD}^{4} 5^{+}$leukocytes in paired blood and BALF were analyzed by flow cytometry 
764

765

766

767

768

769

770

771

772

773

774

775

776

777

778

779

780

781

782

783

784

785

786

and presented. Patients' ID. are presented at the bottom. DPS: days post symptom onset. c and d. Kinetic changes of the indicated leukocytes in respiratory specimens are presented. All the data $(n=49)$ from H\&E staining and flow cytometry are plotted in c. The lines show the kinetic trend of each leukocyte subset, using smoothing splines. Kinetic changes in relative frequencies of the indicated leukocyte subsets in mild (blue, $n=20$ ) and severe (red, $n=29$ ) groups are presented in d. DPS: days post symptom onset.

\section{Fig. 3. Kinetic profiling of inflammatory mediators derived from inflammatory leukocytes} in respiratory specimens. Kinetic changes of inflammatory mediators or biomarkers derived from neutrophils (a), eosinophils (b), mast cells (c), macrophages (d), and cytotoxic T cells/NK cells (e) in respiratory specimens are presented. Concentrations of inflammatory markers in mild (M) and severe (S) cases are also compared and presented as violin plots (right graph, black line: median). The lines show the kinetic trend, using smoothing splines. LCN: lipocalin-2 ( $n=18$ for mild and 23 for severe cases), CALP: calprotectin ( $n=11$ for mild and 19 for severe cases), EDN: eosinophil-derived neurotoxin ( $n=18$ for mild and 23 for severe cases), ECP: eosinophilic cationic protein ( $n=11$ for mild and 19 for severe cases), MCT: mast cell tryptase ( $n=18$ for mild and 23 for severe cases). sCD163 and granzyme A ( $n=18$ for mild and 23 for severe cases). DPS: days post symptom onset. *: $p<0.05$ (two-tailed Mann-Whitney test).

\section{Fig. 4. Correlation of respiratory levels of cytokines, chemokines, and inflammatory} mediators in COVID-19 patients. a. Correlation matrix of 28 inflammatory proteins detected in respiratory samples from COVID-19 patients (see raw data in Supplementary Table S3). The areas of the circles show the value of corresponding correlation coefficients as calculated by the 
Spearman method. Positive correlations are displayed in blue and negative correlations in red. The size of the circle and intensity of color are proportional to correlation coefficients ( 0 to 1 for positive coefficient and 0 to -1 for negative coefficient). The legend to the right of the matrix shows the Spearman correlation coefficients for the corresponding colors. The correlation coefficients of proteins were clustered based on hierarchical clustering. Boxes indicate groups of cytokines with higher correlations. ${ }^{*}: p<0.05, * *: p<0.01, * * *: p<0.001$. b. Kinetic changes of IL-6 and TGF- $\beta$, cytokines showing significant difference between mild (blue, M, $n=11$ ) and severe (red, $\mathrm{S}, n=20$ ) groups. The lines show the kinetic trend, using smoothing splines. Concentrations of the cytokines in mild and severe were also compared and presented as violin plots (right graph, black line: median). DPS: days post symptom onset.

\section{Fig. 5. Kinetic changes of SARS-CoV-2 N-specific antibodies and C3a in respiratory} specimens. Kinetic changes in specific antibody responses against viral $\mathrm{N}$ protein (a) and $\mathrm{C} 3 \mathrm{a}$ (b) in respiratory samples are presented. Specific isotypes of the antigen-specific antibodies are denoted. Blue: mild ( $n=13$ for antibodies and 20 for $\mathrm{C} 3 \mathrm{a}$ ), red: severe ( $n=28$ for antibodies and 20 for $\mathrm{C} 3 \mathrm{a}$ ). The lines show the kinetic trend, using smoothing splines. The levels in mild and severe cases were also compared and presented as violin plots (right side, black line: median). *: $p<0.05$ (Two-tailed Mann-Whitney test). DPS: days post symptom onset. c. Correlation of C3a levels with $\mathrm{N}$-specific IgM (left) and IgG (right) were assessed by linear regression (dashed line) and Spearman's rank test. $p$ values are indicated within the graphs. $n=27$.

Fig. 6. Kinetic changes of SARS-CoV-2 N-specific antibodies and C3a in plasma. Kinetic changes in specific antibody responses against viral $\mathrm{N}$ protein (a), C3a and $\mathrm{C} 5 \mathrm{a}$ (b) in plasma 
samples are presented. Specific isotypes of the antigen-specific antibodies are denoted. Blue: mild $(n=30)$, red: severe $(n=19)$. The lines show the kinetic trend, using smoothing splines. The levels in mild and severe cases were also compared and presented as violin plots (right side, black line: median). DPS: days post symptom onset. ${ }^{*}: p<0.05,{ }^{* *}: p<0.01, * * *: p<0.001$ (Two-tailed Mann-Whitney test). c. Correlation of C5a levels with N-specific IgG1 (left) and IgG3 (right) were assessed by linear regression (dashed line) and Spearman's rank test. $p$ values are indicated within the graphs. $n=61$.

\section{Fig. 7. Kinetic changes of viral loads, inflammatory indicators, antibody responses, and} pulmonary histopathology in a fatal COVID-19 case. a. Kinetic changes of viral loads were evaluated in respiratory specimens in upper respiratory (U.R.) and lower respiratory (L.R.) tract (black dashed line: cut-off value). Kinetic change of CRP in plasma is also presented (red dashed line: upper limit of normal range, $10 \mathrm{mg} / \mathrm{dL}$ ). b. Kinetic changes of lactate dehydrogenase (LDH, red) and creatine phosphokinase (CPK, blue) in plasma. Blue and red dashed lines indicates upper limit of normal ranges for CPK (200 U/L) and LDH (350 U/L), respectively. Microscopic findings in H\&E-stained lung tissues from a fatal case, P15, obtained at D36 (c) and D48 (d) after symptom onset. At a scan view, subpleural lung parenchyma with fibrosis and fibrin deposits are observed (upper left, 12.5x magnification). Higher power views of each representative area (rectangles) are displayed. c. Fibrinous exudate, hemorrhage and congestion in subpleural lung parenchyma are observed (upper right, 200x). A few alveolar macrophages and hemorrhage in intra-alveolar spaces (black arrow) and some lymphoplasmacytic infiltration and smooth muscle proliferation in dense fibrotic interstitium are observed (lower left, 200x). A few neutrophilic infiltration (red arrows) and congested blood vessels reminiscent of granulation 
tissue are noted (lower right, 400x). d. Marked intra-alveolar hemorrhage and congestion in blood vessels are observed along with interstitial thickening and fibrosis (upper right, 200x). In non-hemorrhagic areas, interstitial thickening and fibrosis, alveolar bronchiolization (black arrows), intra-alveolar seromucinous fluid accumulation and variable-sized alveolar macrophages (asterisks) are observed. Infiltration of a few lymphoid cells and plasma cells in interstitium is noted (blue arrow) (lower left, 200x). Alveolar bronchiolization (bronchiolar metaplasia of alveoli) (black arrows), detachment of alveolar epithelial cells (green arrows) and interstitial thickening and fibrosis (asterisks) are observed (lower right, 200x). e. Kinetic changes in specific antibody responses against viral $\mathrm{N}$ protein and $\mathrm{C} 5 \mathrm{a}$ in respiratory samples (left) and plasma (right). f. Localization of SARS-CoV-2 N antigens in lung tissues. Representative images of triple immunofluorescence-stained sections of lung tissues obtained from the fatal case at D36 (upper panels) and D48 (lower panels). Immunofluorescence for endothelial marker (CD31, green, left panels) or epithelial marker (CK: pan-cytokeratin, green, right panels), together with SARS-CoV-2 N antigens (red), are indicated. Nuclear DNA was counterstained with 4,6diamidino-2-phenylindole (blue). Differential interference contrast (DIC) images show lung parenchyma. Blood vessels are indicated by white asterisks. Scale bar, $100 \mu \mathrm{m}$.

\section{Fig. 8. Pathologic association of complement activation and immune complexes deposition} as identified by immunohistochemistry and immunofluorescence analysis of pulmonary autopsy samples from a fatal COVID-19 case. a. Immunohistochemical detection of C5b-9 membrane attack complexes in pulmonary parenchyma using lung tissues from a fatal case obtained at D36 (left panels) and D48 (right panels). C5b-9 staining was observed in media layer of vascular walls (asterisks), airway epithelial cells (purple arrows), and inflammatory cells such 
856

857

858

859

860

861

862

863

864 $865 \quad 100 \mu \mathrm{m}$.

866

867

868

869

870

871

872

873

874

875

876

877

as macrophages (green arrows) and lymphoplasma cells (red arrows). Scale bar: $100 \mu \mathrm{m} . \mathbf{b}$.

Depositions of IgG immune complexes in airways and blood vessels were identified by immunofluorescence analysis. Representative immunofluorescence images of lung tissues obtained from the fatal case at D36 (upper panels) and D48 (lower panels) are presented. Immunofluorescence of epithelial marker (CK: pan-cytokeratin, green, right panels), SARSCoV-2 N antigens (red), and IgG (white) are indicated. White arrows indicate depositions of IgG immune complexes in the luminal spaces, hyaline membranes, and fibrin deposits. Nuclear DNA was counterstained with 4,6-diamidino-2-phenylindole (blue). Differential interference contrast (DIC) images show lung parenchyma. Blood vessels are indicated by white asterisks. Scale bar,

Fig. 9. Enhanced gene set signatures for $F c \gamma R$ signaling and complement activation in myeloid cells of respiratory samples from severe COVID-19 cases. a. UMAP presentation of major cell types and associated clusters in respiratory leukocytes from COVID-19 patients. Data sets were retrieved from two previous studies. ${ }^{55,56}$ Ep.: epithelial cells, B: B cells, T.NK: T and NK cells, Mo.MF.DC: monocytes, macrophages, and dendritic cells, Neutro.: neutrophils. b. Hallmark gene set scores for inflammatory response, computed for the indicated leukocyte subsets (all patients' samples combined), were compared with those of healthy controls (HC). $p$ values for differences between HC and COVID-19 patients as assessed by two-tailed MannWhitney $\mathrm{U}$ test are presented. c. Computed hallmark gene set activity scores of Fc $\gamma \mathrm{R}$ signaling and complement activation, for the indicated myeloid subsets. $P$ values for differences among HC and COVID-19 patients with mild (M) or severe (S) symptoms, as assessed by two-tailed 
878 Mann-Whitney U test, are presented.

879

880

881

882

883

884

885

886

887

888

889

890

891

892

893

894

895

896

897

898

899

900 
901

902

903

904

905

906

907

908

909

910

911

912

913

914

915

916

917

918

919

920

921

922

\section{Supplementary Information}

Table S1. Baseline characteristics of COVID-19 patients included in this study.

Table S2. Summary of cytological analysis of sputa and BALFs by hematoxylin and eosin (H\&E) staining.

Table S3. Raw data of 28 cytokines, chemokines, and inflammatory mediators measured in respiratory specimens collected from COVID-19 patients.

Fig. S1. Clinical courses of group 1 patients with COVID-19 enrolled in the present study.

Fig. S2. Gating strategy for the characterization of leukocytes in blood and BALFs.

Fig. S3. Relative frequencies of myeloid cell subtypes in blood and BALF specimens from patients with severe COVID-19. The frequencies are percentile among CD45 ${ }^{+}$leukocytes.

Fig. S4. Relative frequencies of lymphoid cell subtypes in blood and BALF specimens from patients with severe COVID-19. The frequencies are percentile among lymphocytes.

Fig. S5. Relative frequencies of $\mathrm{T}$ and NKT cell subtypes in blood and BALF specimens from patients with severe COVID-19. The frequencies are percentile among lymphocytes.

Fig. S6. Kinetic changes of 28 cytokines, chemokines, and inflammatory mediators measured in respiratory specimens collected from COVID-19 patients. Raw data for each graph are presented in Supplementary Table S3. The purple lines show the kinetic trend of each inflammatory marker, using smoothing splines. Blue dots: mild cases, red dots: severe cases. n.d.: not detected.

Fig. S7. Kinetic changes of specific antibodies against viral N protein in plasma from COVID-19 patients. Specific isotypes of the antigen-specific antibodies are indicated. Blue are from eight 
923 mild patients and red are from five severe cases.

924 Fig. S8. Kinetics of complete blood counts in blood collected from COVID-19 patients. Normal 925 range of each leukocyte count is indicated as gray background.

926 Fig. S9. Depositions of IgG immune complexes in lung tissue were identified by 927 immunofluorescence analysis. Representative immunofluorescence images of lung tissue 928 obtained from the fatal case at D48 are presented. Immunofluorescence of SARS-CoV-2 N 929 antigens (red) and IgG (white) are indicated. Blue: nuclei, DIC: Differential interference contrast, 930 scale bar: $100 \mu \mathrm{m}$. 
Figures
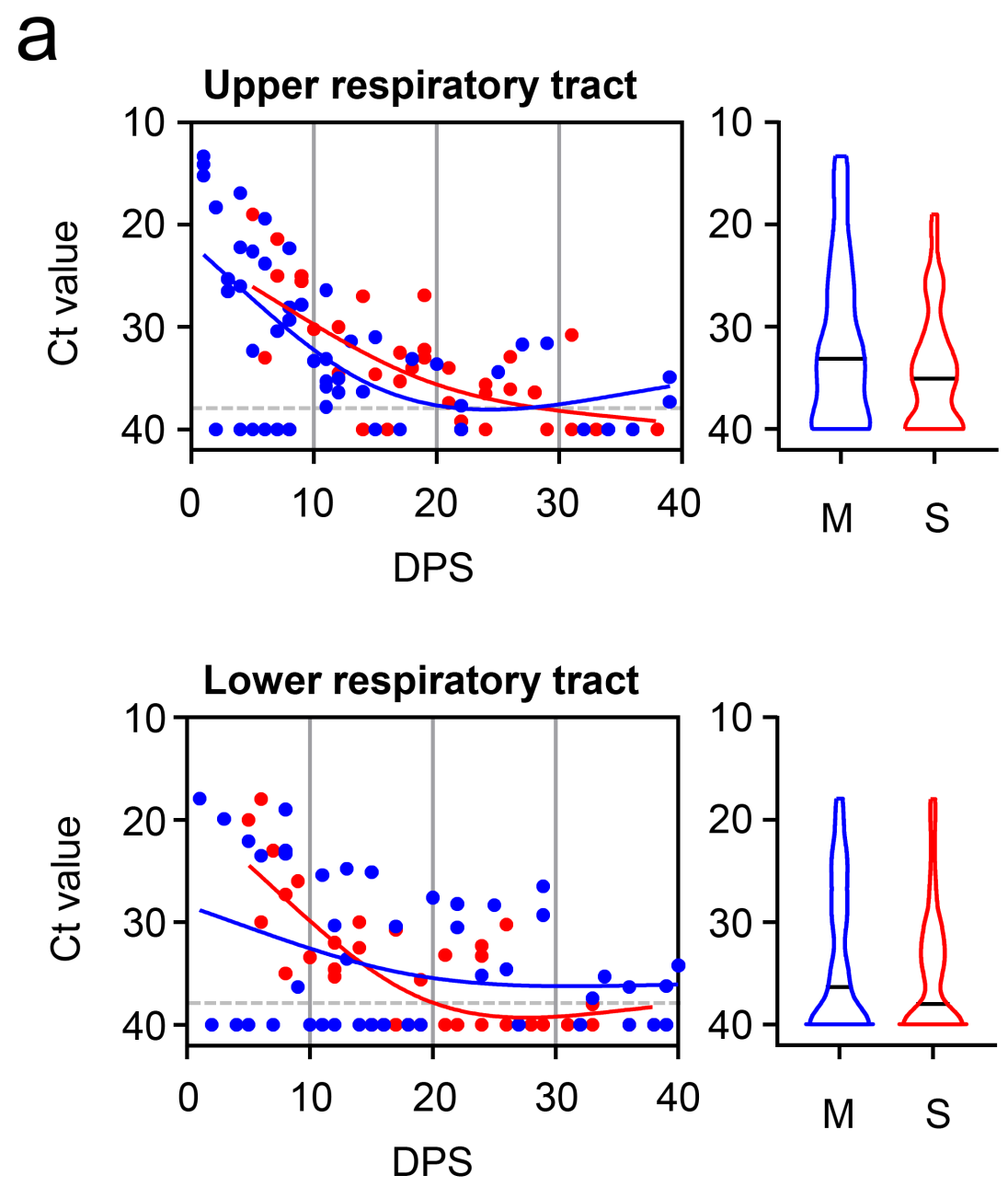

b
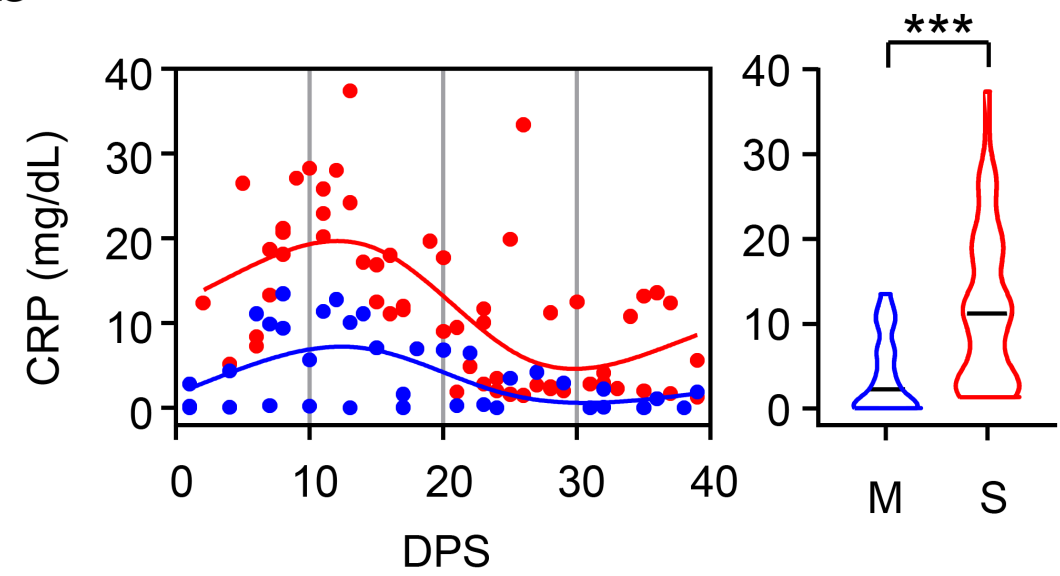

Figure 1

Kinetic changes of respiratory viral loads and C-reactive proteins in plasma. a. Kinetic changes of viral loads were evaluated in respiratory specimens (nasopharygeal/throat swabs for upper respiratory tract and sputa for lower respiratory tract) by quantitative RT-PCR targeting the E gene of SARS-CoV-2 and 
presented as $\mathrm{Ct}$ values. Blue circles ( $\mathrm{n}=49$ and 41 for upper and lower respiratory specimens, respectively) are from eight mild patients and red circles ( $n=38$ and 39 for upper and lower respiratory specimens, respectively) are from five severe cases. The blue and red lines show the trend in viral load, using smoothing splines (dashed line: cut-off value). Distribution of Ct values in mild (M) and severe (S) are also presented as violin plots (right graph, black line: median). b. Kinetic change of C-reactive proteins (CRP) in plasma is presented. Blue circles are from nine mild patients $(n=37)$ and red circles are from five severe cases $(n=23)$. Distribution of CRP levels in mild $(M)$ and severe $(S)$ are also presented as violin plots (right graph, black line: median). DPS: days post symptom onset. $* \star *$ : $p<0.001$ (Two-tailed Mann-Whitney test). 
a

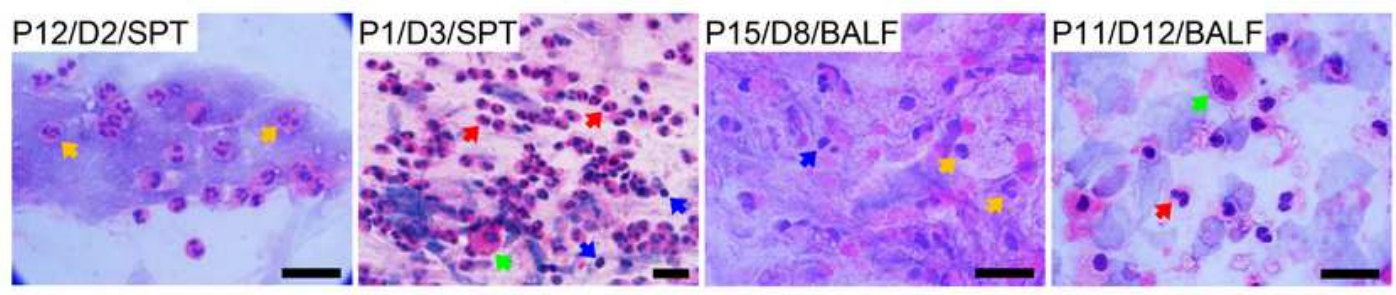

b
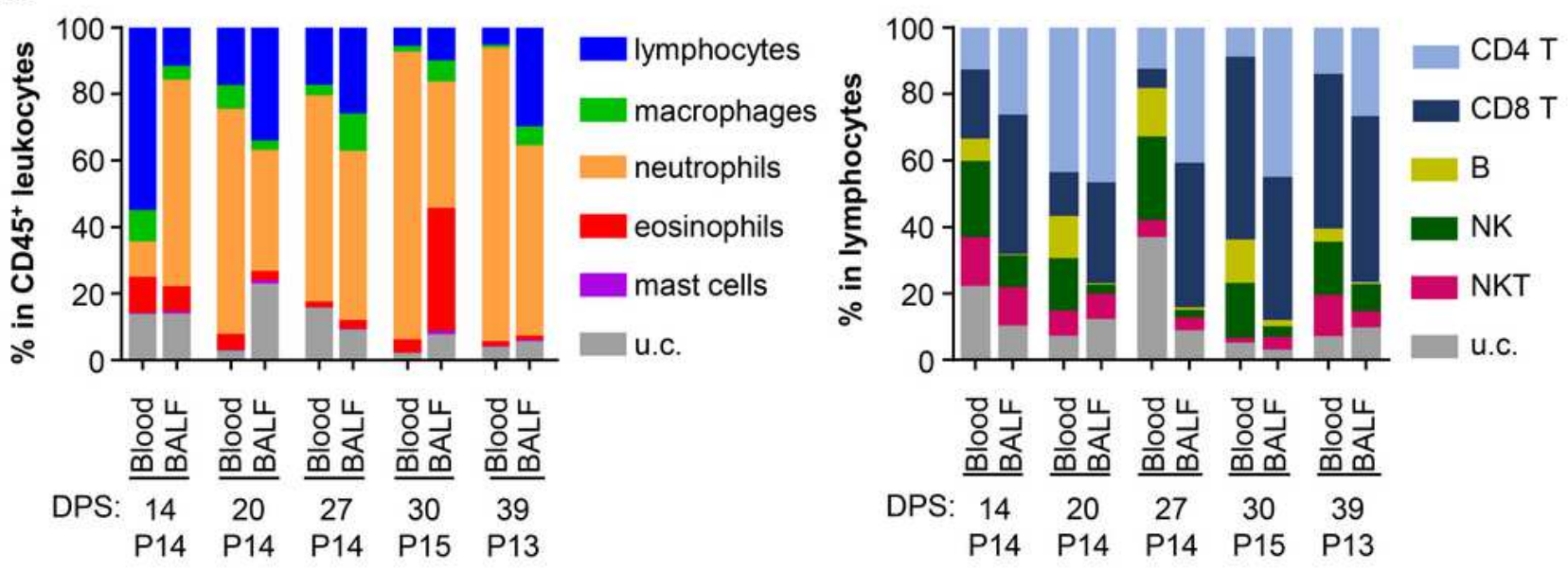

C

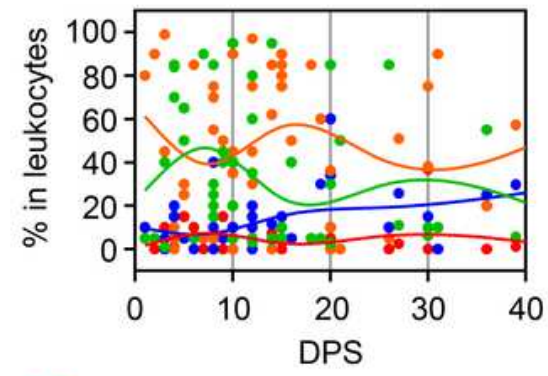

- Macrophages

$\rightarrow$ PMN

- Eosinophils

- Lymphocytes d

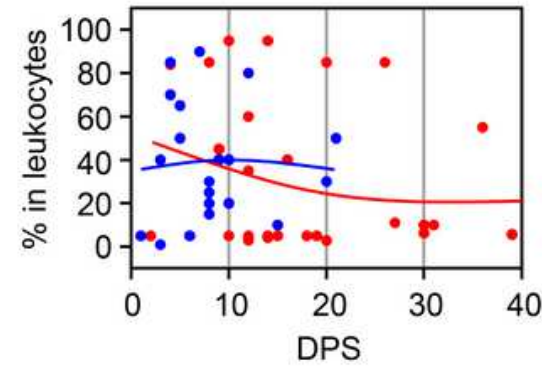

Lymphocytes

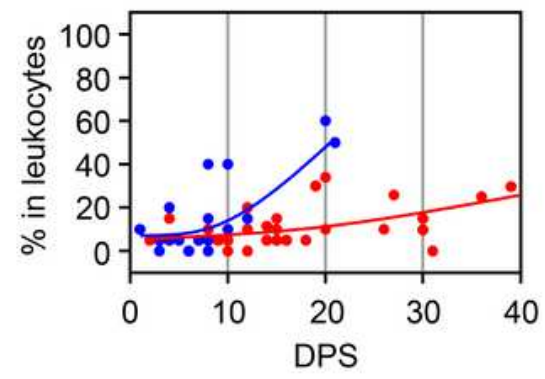

PMNs

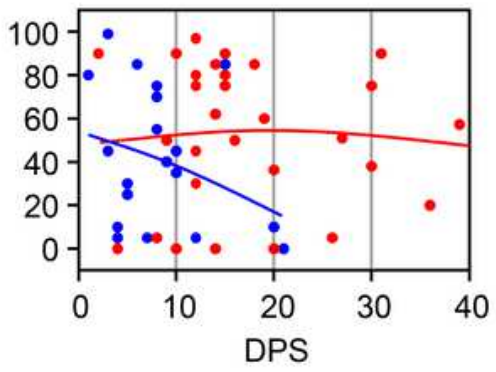

Eosinophils

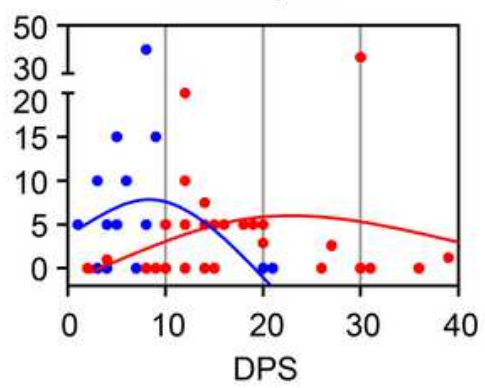

Figure 2

Kinetic changes in respiratory specimens collected from COVID-19 patients. a. Representative images of cytological analysis of sputa and BALFs by hematoxylin and eosin (H\&E) staining. The patient's ID., collection day after symptom onset, and types of specimens (SPT: sputum, and BALF: bronchoalveolar lavage fluid) are presented in the upper left text box. Arrows indicate PMNs (orange, mostly neutrophils), eosinophils (red), lymphocytes (blue), and macrophages (green). Scale bar: $20 \mu \mathrm{m}$. b. Relative 
frequencies of the indicated leukocyte subsets among CD45+ leukocytes in paired blood and BALF were analyzed by flow cytometry and presented. Patients' ID. are presented at the bottom. DPS: days post symptom onset. $c$ and d. Kinetic changes of the indicated leukocytes in respiratory specimens are presented. All the data $(n=49)$ from H\&E staining and flow cytometry are plotted in $c$. The lines show the kinetic trend of each leukocyte subset, using smoothing splines. Kinetic changes in relative frequencies of the indicated leukocyte subsets in mild (blue, $n=20)$ and severe $(r e d, n=29)$ groups are presented in $d$. DPS: days post symptom onset.
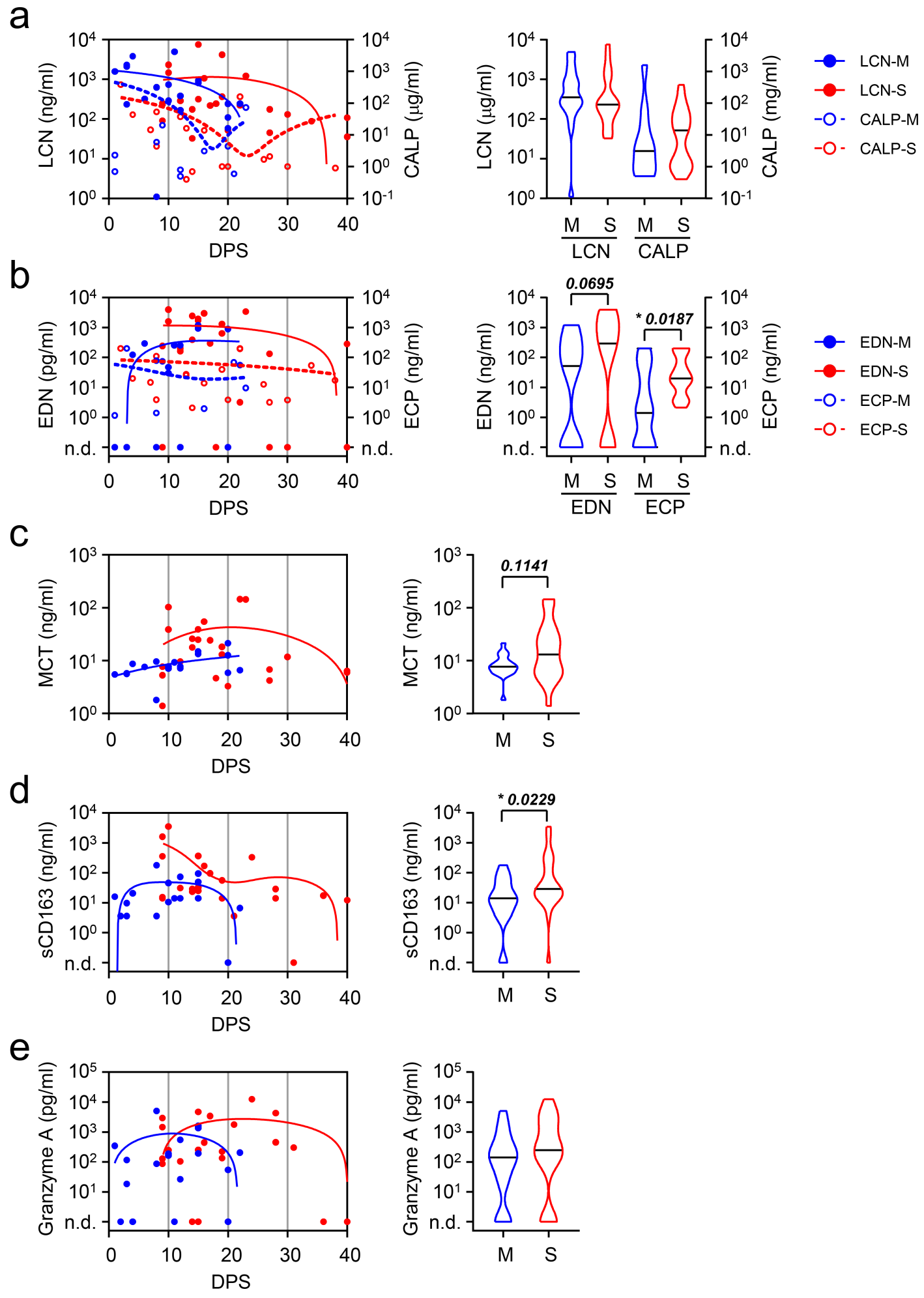


\section{Figure 3}

Kinetic profiling of inflammatory mediators derived from inflammatory leukocytes in respiratory specimens. Kinetic changes of inflammatory mediators or biomarkers derived from neutrophils (a), eosinophils (b), mast cells (c), macrophages (d), and cytotoxic T cells/NK cells (e) in respiratory specimens are presented. Concentrations of inflammatory markers in mild (M) and severe (S) cases are also compared and presented as violin plots (right graph, black line: median). The lines show the kinetic trend, using smoothing splines. LCN: lipocalin-2 ( $n=18$ for mild and 23 for severe cases), CALP: calprotectin ( $\mathrm{n}=11$ for mild and 19 for severe cases), EDN: eosinophil-derived neurotoxin ( $\mathrm{n}=18$ for mild and 23 for severe cases), ECP: eosinophilic cationic protein ( $n=11$ for mild and 19 for severe cases), MCT: mast cell tryptase ( $n=18$ for mild and 23 for severe cases). sCD163 and granzyme $A$ ( $n=18$ for mild and 23 for severe cases). DPS: days post symptom onset. *: $p<0.05$ (two-tailed Mann-Whitney test). 
a

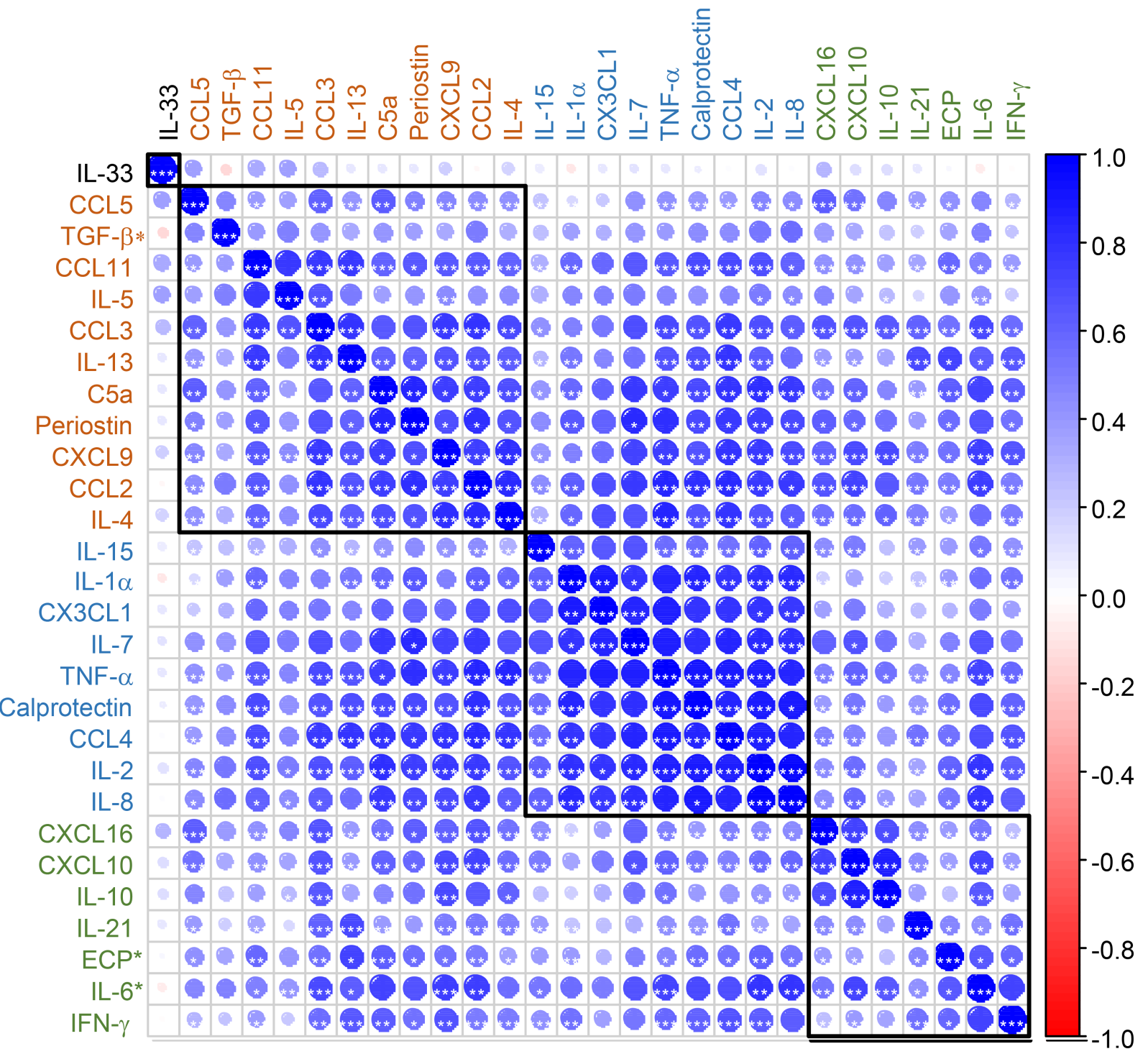

b
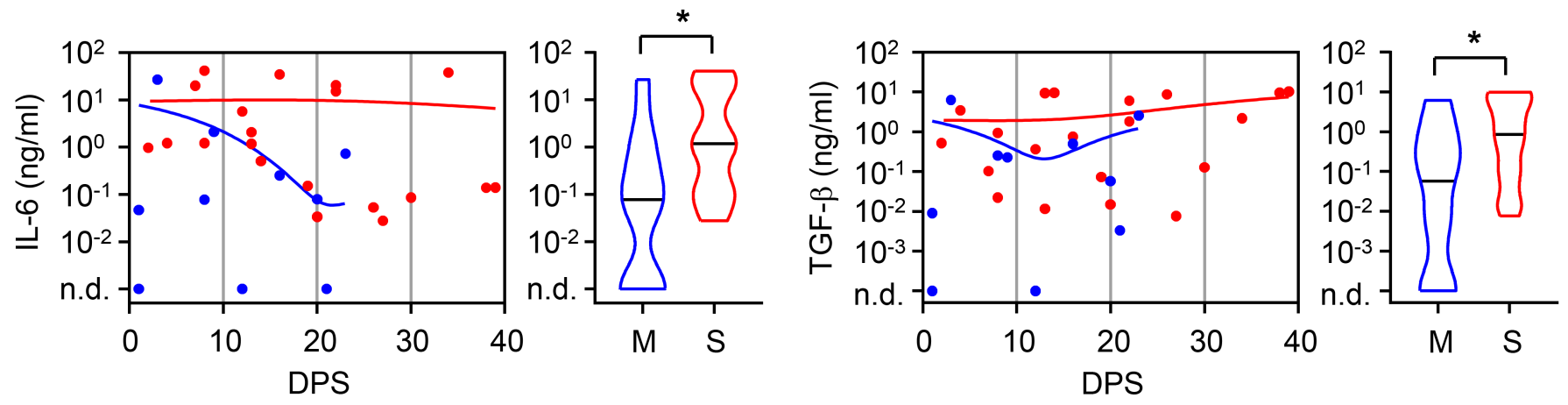

Figure 4

Correlation of respiratory levels of cytokines, chemokines, and inflammatory mediators in COVID-19 patients. a. Correlation matrix of 28 inflammatory proteins detected in respiratory samples from COVID19 patients (see raw data in Supplementary Table S3). The areas of the circles show the value of corresponding correlation coefficients as calculated by the Spearman method. Positive correlations are displayed in blue and negative correlations in red. The size of the circle and intensity of color are 
proportional to correlation coefficients ( 0 to 1 for positive coefficient and 0 to -1 for negative coefficient). The legend to the right of the matrix shows the Spearman correlation coefficients for the corresponding colors. The correlation coefficients of proteins were clustered based on hierarchical clustering. Boxes indicate groups of cytokines with higher correlations. ${ }^{*}: p<0.05, * *: p<0.01,{ }^{* * *}: p<0.001$. b. Kinetic changes of IL- 6 and TGF- $\beta$ cytokines showing significant difference between mild (blue, $M, n=11$ ) and severe (red, $S, n=20$ ) groups. The lines show the kinetic trend, using smoothing splines. Concentrations of the cytokines in mild and severe were also compared and presented as violin plots (right graph, black line: median). DPS: days post symptom onset.
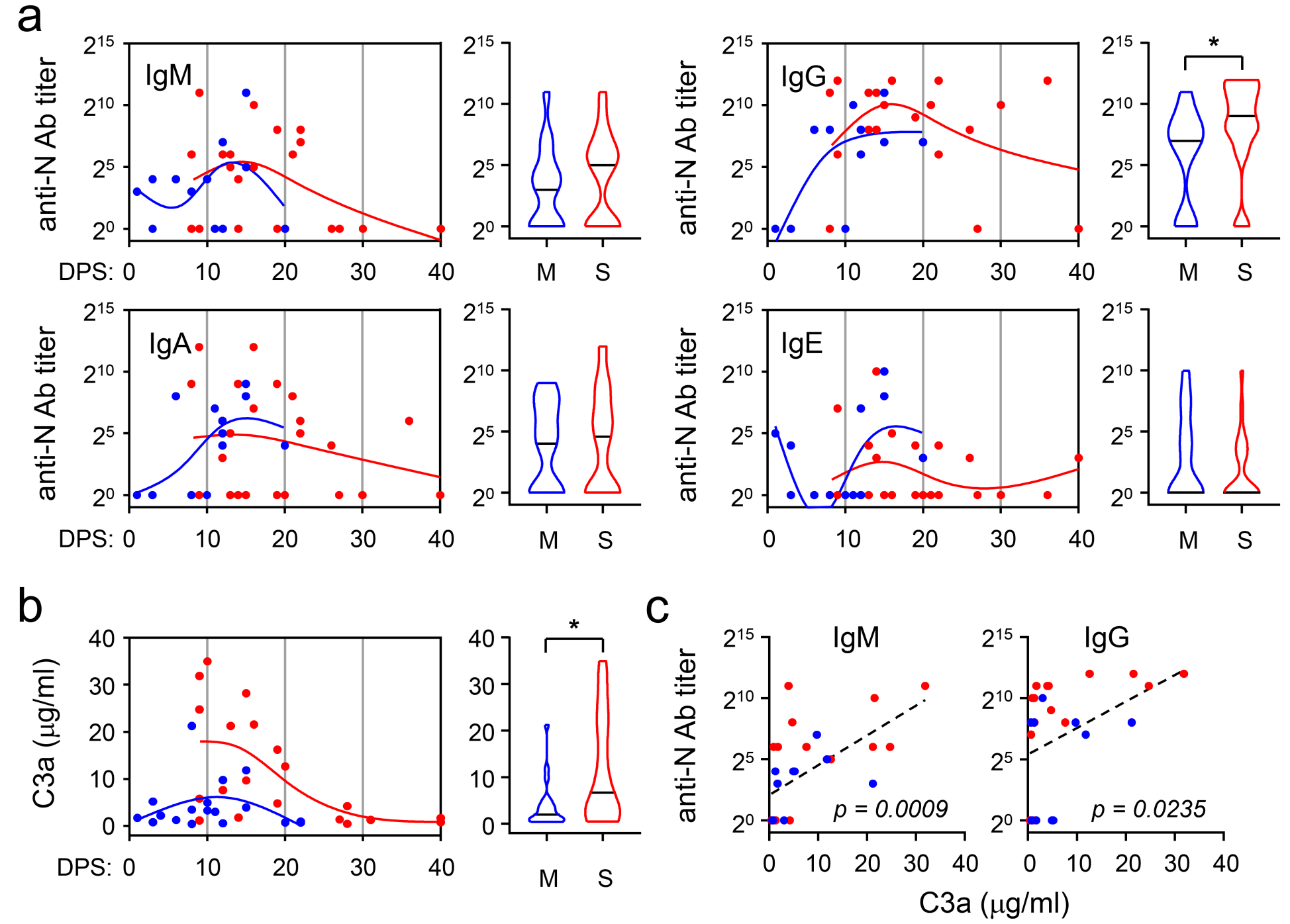

Figure 5

Kinetic changes of SARS-CoV-2 N-specific antibodies and C3a in respiratory specimens. Kinetic changes in specific antibody responses against viral N protein (a) and C3a (b) in respiratory samples are presented. Specific isotypes of the antigen-specific antibodies are denoted. Blue: mild ( $n=13$ for antibodies and 20 for $\mathrm{C} 3 \mathrm{a}$ ), red: severe ( $\mathrm{n}=28$ for antibodies and 20 for $\mathrm{C} 3 \mathrm{a})$. The lines show the kinetic trend, using smoothing splines. The levels in mild and severe cases were also compared and presented as violin plots (right side, black line: median). *: $p<0.05$ (Two-tailed Mann-Whitney test). DPS: days post 
symptom onset. c. Correlation of C3a levels with N-specific lgM (left) and IgG (right) were assessed by linear regression (dashed line) and Spearman's rank test. $\mathrm{p}$ values are indicated within the graphs. $\mathrm{n}=27$.
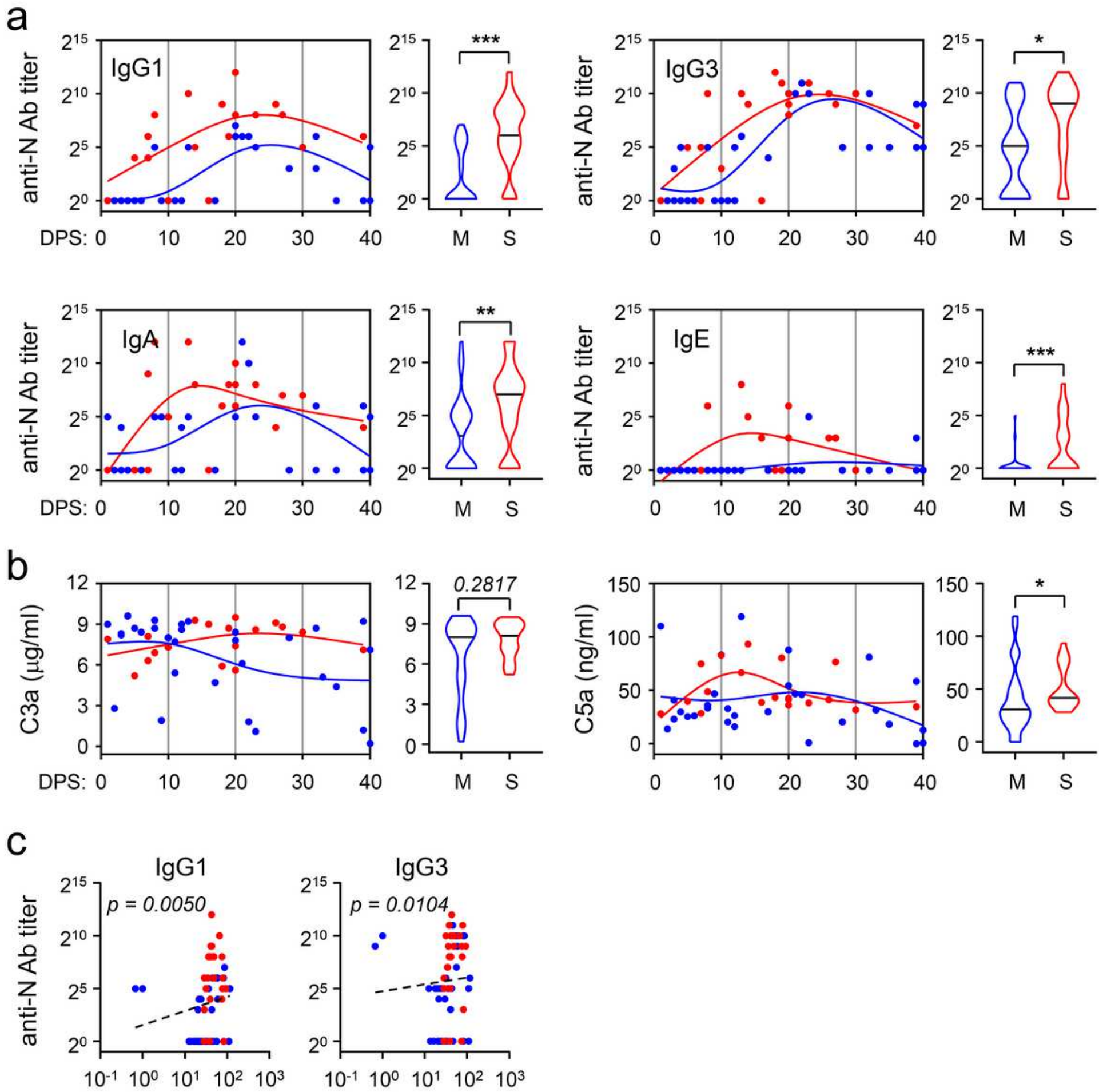

$\mathrm{C} 5 \mathrm{a}(\mathrm{ng} / \mathrm{ml})$

Figure 6

Kinetic changes of SARS-CoV-2 N-specific antibodies and C3a in plasma. Kinetic changes in specific antibody responses against viral $\mathrm{N}$ protein (a), C3a and C5a (b) in plasma samples are presented. Specific isotypes of the antigen-specific antibodies are denoted. Blue: mild $(n=30)$, red: severe $(n=19)$. 
The lines show the kinetic trend, using smoothing splines. The levels in mild and severe cases were also compared and presented as violin plots (right side, black line: median). DPS: days post symptom onset. * $: p<0.05,{ }^{* *}: p<0.01, * * *: p<0.001$ (Two-tailed Mann-Whitney test). c. Correlation of C5a levels with Nspecific lgG1 (left) and IgG3 (right) were assessed by linear regression (dashed line) and Spearman's rank test. $p$ values are indicated within the graphs. $n=61$.

a

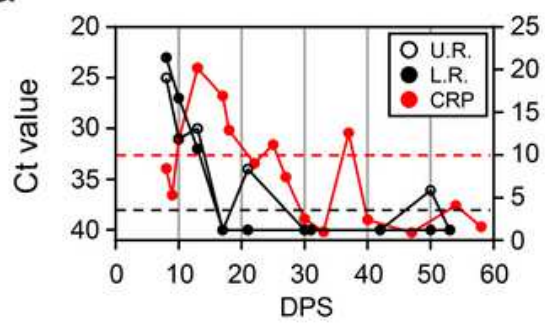

C

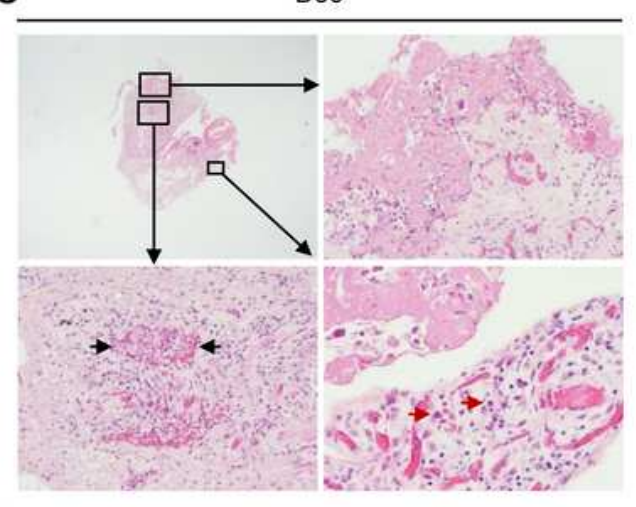

e

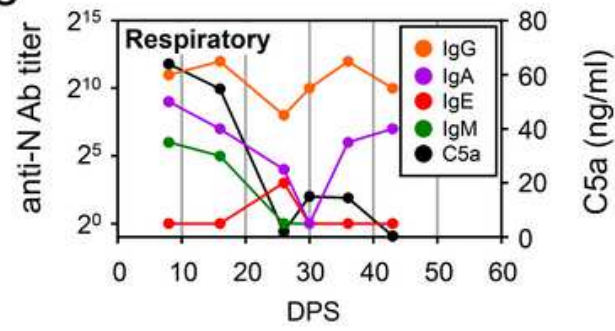

f
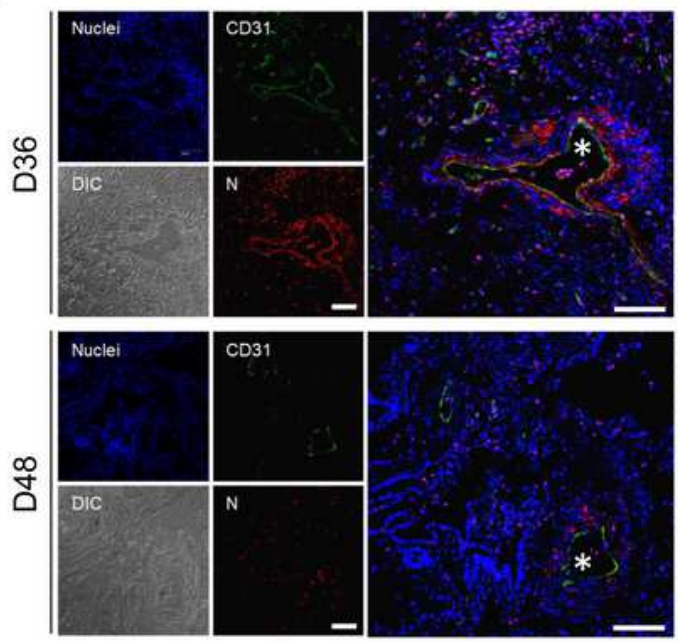

b

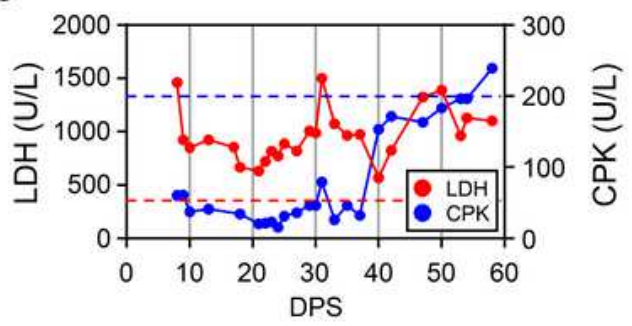

d
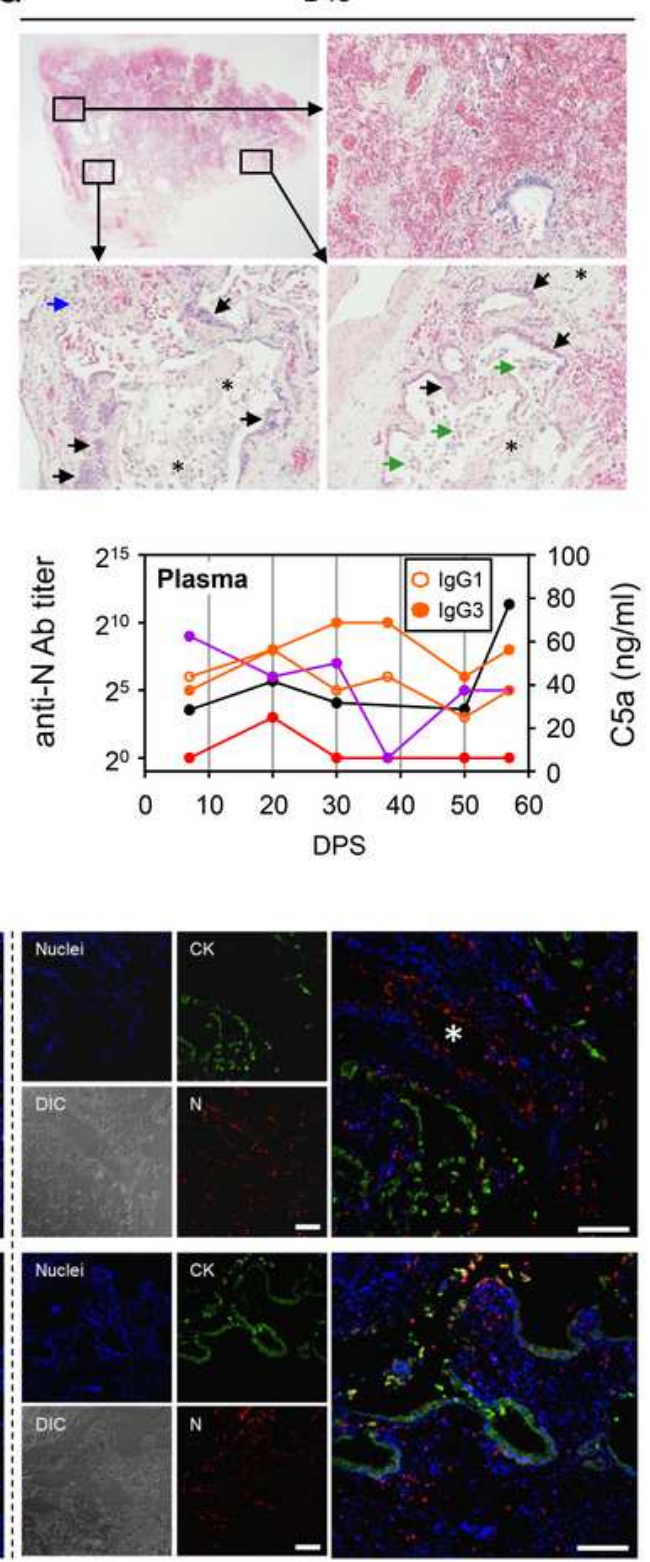

Figure 7 
Kinetic changes of viral loads, inflammatory indicators, antibody responses, and pulmonary histopathology in a fatal COVID-19 case. a. Kinetic changes of viral loads were evaluated in respiratory specimens in upper respiratory (U.R.) and lower respiratory (L.R.) tract (black dashed line: cut-off value). Kinetic change of CRP in plasma is also presented (red dashed line: upper limit of normal range, 10 $\mathrm{mg} / \mathrm{dL}$ ). b. Kinetic changes of lactate dehydrogenase ( $\mathrm{LDH}$, red) and creatine phosphokinase (CPK, blue) in plasma. Blue and red dashed lines indicates upper limit of normal ranges for CPK (200 U/L) and LDH (350 U/L), respectively. Microscopic findings in H\&E-stained lung tissues from a fatal case, P15, obtained at D36 (c) and D48 (d) after symptom onset. At a scan view, subpleural lung parenchyma with fibrosis and fibrin deposits are observed (upper left, 12.5x magnification). Higher power views of each representative area (rectangles) are displayed. c. Fibrinous exudate, hemorrhage and congestion in subpleural lung parenchyma are observed (upper right, 200x). A few alveolar macrophages and hemorrhage in intra-alveolar spaces (black arrow) and some lymphoplasmacytic infiltration and smooth muscle proliferation in dense fibrotic interstitium are observed (lower left, 200x). A few neutrophilic infiltration (red arrows) and congested blood vessels reminiscent of granulation tissue are noted (lower right, 400x). d. Marked intra-alveolar hemorrhage and congestion in blood vessels are observed along with interstitial thickening and fibrosis (upper right, 200x). In non-hemorrhagic areas, interstitial thickening and fibrosis, alveolar bronchiolization (black arrows), intra-alveolar seromucinous fluid accumulation and variable-sized alveolar macrophages (asterisks) are observed. Infiltration of a few lymphoid cells and plasma cells in interstitium is noted (blue arrow) (lower left, 200x). Alveolar bronchiolization (bronchiolar metaplasia of alveoli) (black arrows), detachment of alveolar epithelial cells (green arrows) and interstitial thickening and fibrosis (asterisks) are observed (lower right, 200x). e. Kinetic changes in specific antibody responses against viral $\mathrm{N}$ protein and $\mathrm{C5a}$ in respiratory samples (left) and plasma (right). f. Localization of SARS-CoV-2 $\mathrm{N}$ antigens in lung tissues. Representative images of triple immunofluorescence-stained sections of lung tissues obtained from the fatal case at D36 (upper panels) and D48 (lower panels). Immunofluorescence for endothelial marker (CD31, green, left panels) or epithelial marker (CK: pan-cytokeratin, green, right panels), together with SARS-CoV-2 N antigens (red), are indicated. Nuclear DNA was counterstained with 4,6-diamidino-2-phenylindole (blue). Differential interference contrast (DIC) images show lung parenchyma. Blood vessels are indicated by white asterisks. Scale bar, $100 \mu \mathrm{m}$. 
a

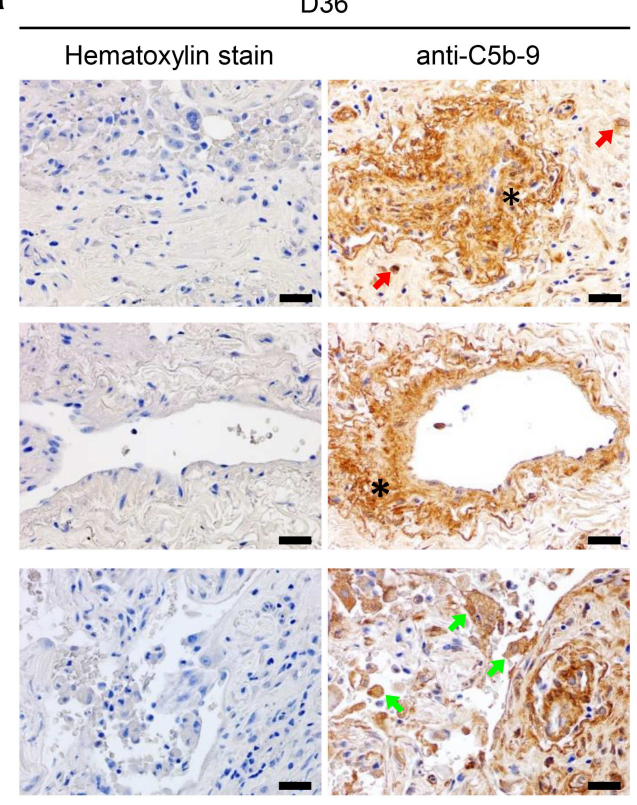

D48
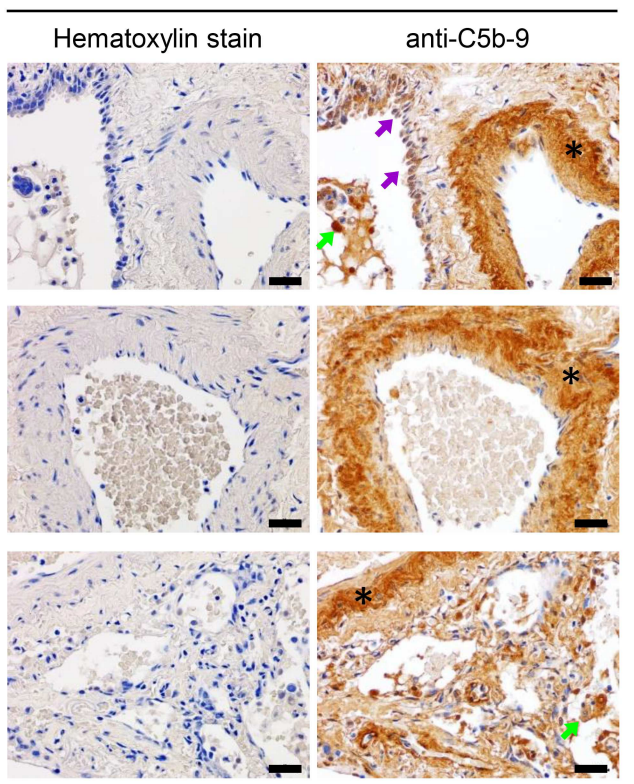

b

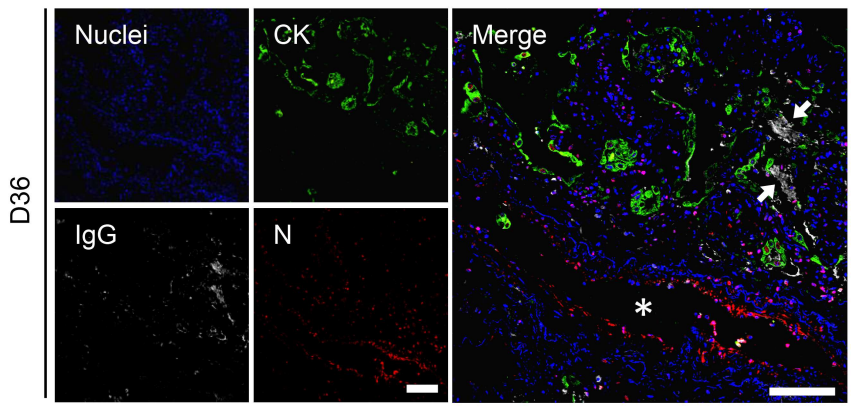

DIC
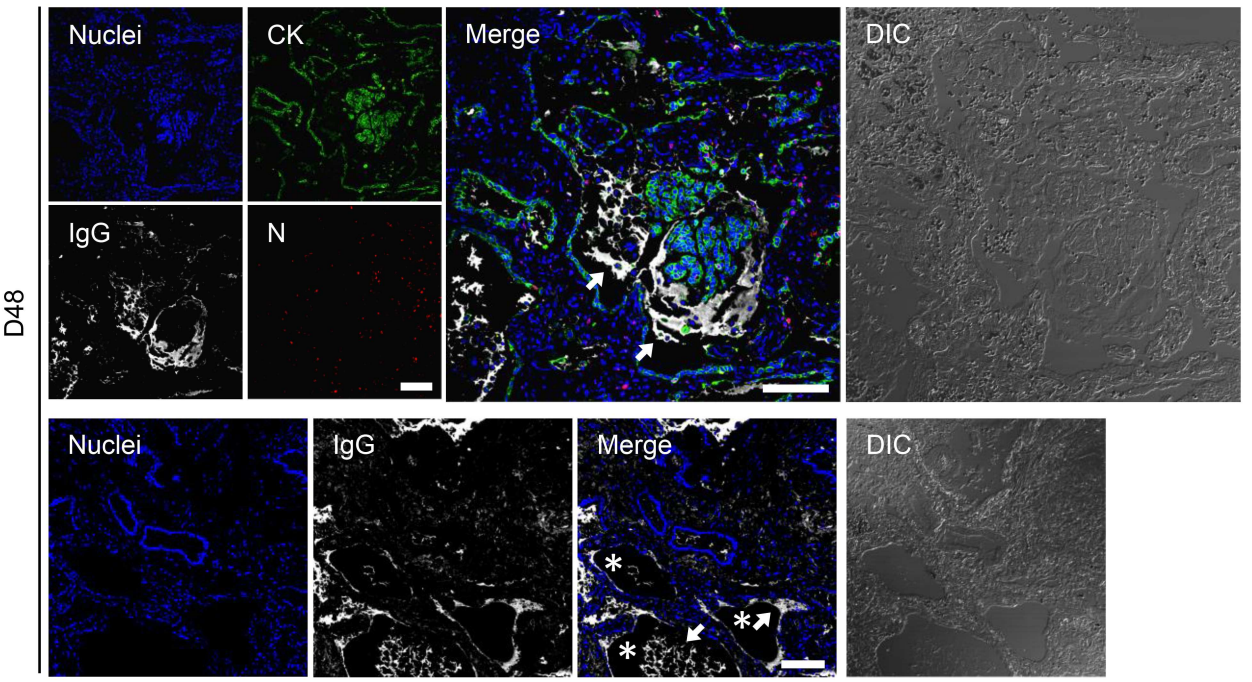

\section{Figure 8}

Pathologic association of complement activation and immune complexes deposition as identified by immunohistochemistry and immunofluorescence analysis of pulmonary autopsy samples from a fatal COVID-19 case. a. Immunohistochemical detection of C5b-9 membrane attack complexes in pulmonary parenchyma using lung tissues from a fatal case obtained at D36 (left panels) and D48 (right panels). C5b-9 staining was observed in media layer of vascular walls (asterisks), airway epithelial cells (purple 
arrows), and inflammatory cells such as macrophages (green arrows) and lymphoplasma cells (red arrows). Scale bar: $100 \mu \mathrm{m}$. b. Depositions of IgG immune complexes in airways and blood vessels were identified by immunofluorescence analysis. Representative immunofluorescence images of lung tissues obtained from the fatal case at D36 (upper panels) and D48 (lower panels) are presented.

Immunofluorescence of epithelial marker (CK: pan-cytokeratin, green, right panels), SARS-CoV-2 N antigens (red), and IgG (white) are indicated. White arrows indicate depositions of IgG immune complexes in the luminal spaces, hyaline membranes, and fibrin deposits. Nuclear DNA was counterstained with 4,6-diamidino-2-phenylindole (blue). Differential interference contrast (DIC) images show lung parenchyma. Blood vessels are indicated by white asterisks. Scale bar, $100 \mu \mathrm{m}$. 

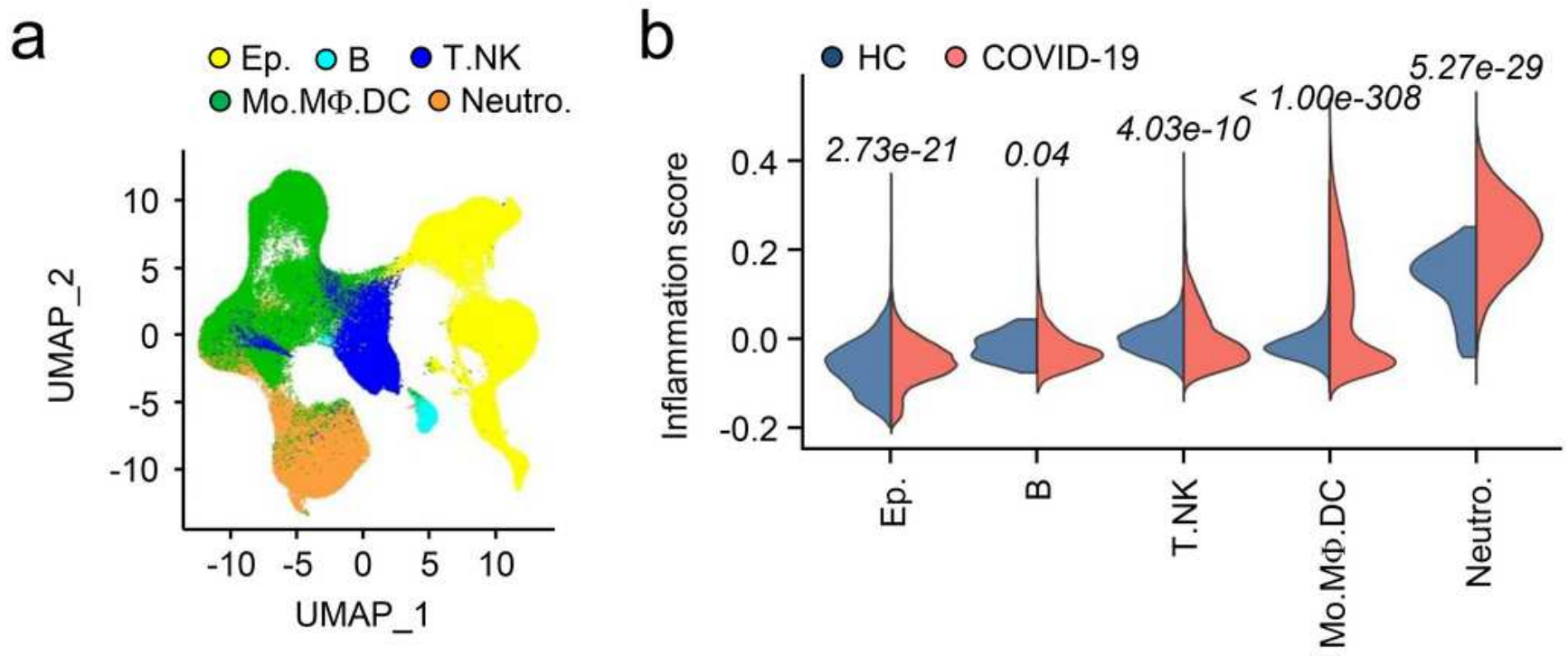

C

Inflammation

FcyR signal

C' activation

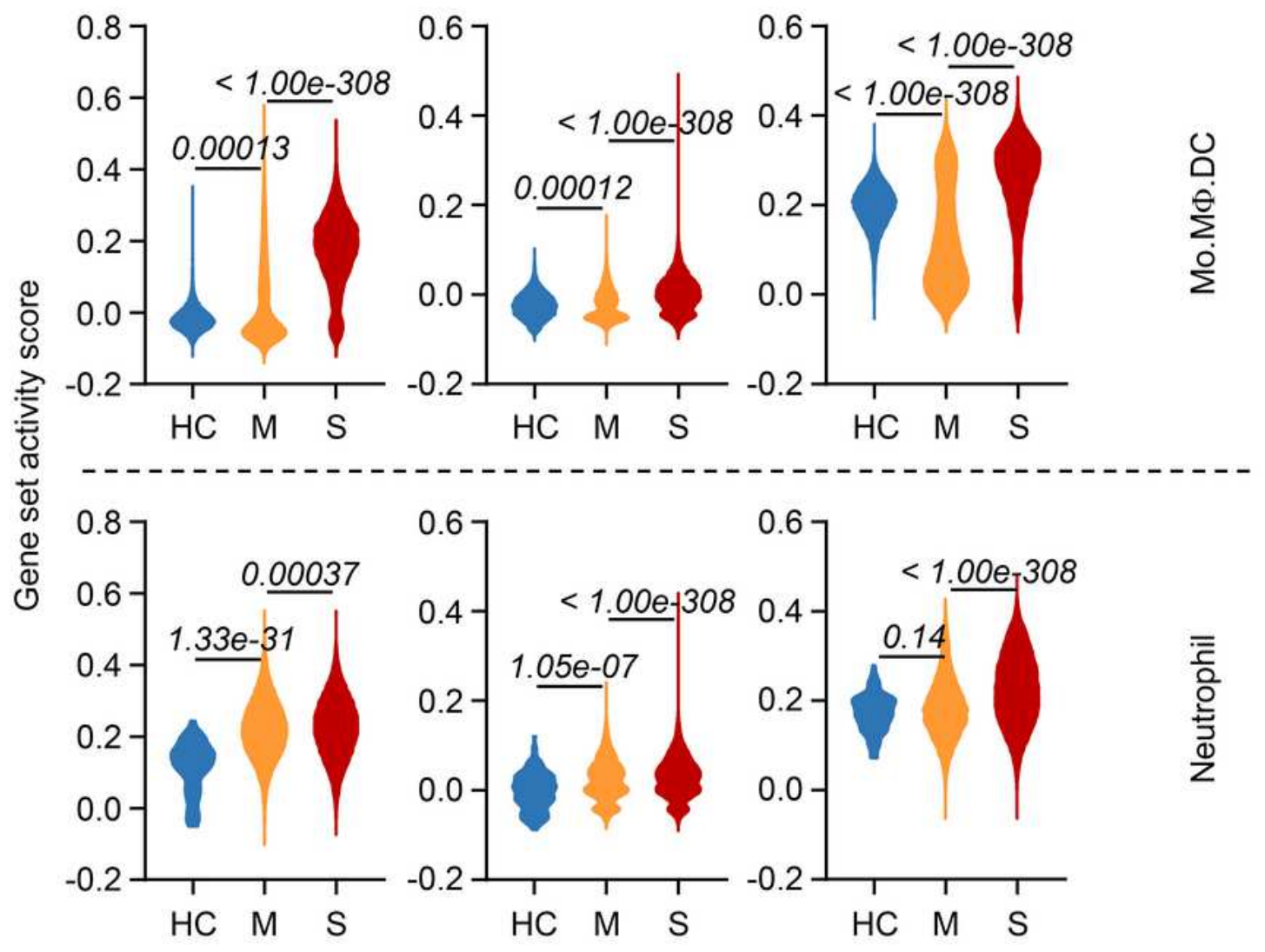

\section{Figure 9}

Enhanced gene set signatures for FcyR signaling and complement activation in myeloid cells of respiratory samples from severe COVID-19 cases. a. UMAP presentation of major cell types and associated clusters in respiratory leukocytes from COVID-19 patients. Data sets were retrieved from two previous studies.55,56 Ep.: epithelial cells, B: B cells, T.NK: T and NK cells, Mo.MF.DC: monocytes, macrophages, and dendritic cells, Neutro.: neutrophils. b. Hallmark gene set scores for inflammatory 
response, computed for the indicated leukocyte subsets (all patients' samples combined), were compared with those of healthy controls (HC). p values for differences between $\mathrm{HC}$ and COVID-19 patients as assessed by two-tailed Mann-Whitney $U$ test are presented. c. Computed hallmark gene set activity scores of FcyR signaling and complement activation, for the indicated myeloid subsets. $P$ values for differences among HC and COVID-19 patients with mild (M) or severe (S) symptoms, as assessed by twotailed Mann-Whitney U test, are presented.

\section{Supplementary Files}

This is a list of supplementary files associated with this preprint. Click to download.

- SupplementaryFigures.pdf

- SupplementaryTableS13.xls 